\title{
Consecutive Patterns: From Permutations to Column-Convex Polyominoes and Back
}

\author{
Don Rawlings \\ Mathematics Department \\ California Polytechnic State University \\ San Luis Obispo, Ca. 93407 \\ drawling@calpoly.edu
}

\author{
Mark Tiefenbruck \\ Mathematics Department \\ University of California \\ San Diego, Ca. 92093 \\ mtiefenb@math.ucsd.edu
}

Submitted: Feb 24, 2010; Accepted: Apr 4, 2010; Published: Apr 19, 2010

Mathematics Subject Classification: 05A15

\begin{abstract}
We expose the ties between the consecutive pattern enumeration problems associated with permutations, compositions, column-convex polyominoes, and words. Our perspective allows powerful methods from the contexts of compositions, columnconvex polyominoes, and of words to be applied directly to the enumeration of permutations by consecutive patterns. We deduce a host of new consecutive pattern results, including a solution to the $(2 m+1)$-alternating pattern problem on permutations posed by Kitaev.
\end{abstract}

Keywords ascents, consecutive pattern, column-convex polyomino, descents, levels, maxima, peaks, twin peaks, up-down type, valleys, variation

\section{Introduction}

The problems of enumerating permutations, compositions, and words by patterns formed by consecutive terms (parts or letters) have been widely studied and, for the most part, their stories are separate and parallel. In contrast, the problem of enumerating columnconvex polyominoes (CCPs) by consecutive patterns has received only scant and indirect consideration.

Our primary purpose is to show that these problem sets are in fact intimately related. More precisely, if $\mathcal{P S}, \mathcal{P C}, \mathcal{P C C P}$, and $\mathcal{P} \mathcal{W}$ respectively denote the sets of consecutive pattern enumeration problems on permutations, compositions, column-convex polyominoes, and words, then

$$
\mathcal{P S} \subset \mathcal{P C} \subset \mathcal{P C C P} \subset \mathcal{P} \mathcal{W}
$$

The significance of (1) is that it allows powerful methods from the larger problem sets to be applied to the smaller problem sets. To illustrate, we will show how various 
results on words as well as Bousquet-Mélou's [4] adaptation of Temperley's [37] method for enumerating CCPs may be used to count permutations by consecutive patterns.

In particular, we exploit the perspective of (1) to $q$-count permutations by $(i, d)$-peaks, up-down type, uniform $m$-peak ranges, and $(i, m)$-maxima. Notably, a specialization of Corollary 4 provides a solution to the $(2 m+1)$-alternating pattern problem on permutations posed by Kitaev [25, Problem 1]. We will also show that the generating function for permutations by a given pattern is deducible from the generating function for a related pattern permutation set; for instance, the generating function for permutations by peaks may be obtained from the one for up-down permutations of odd length.

Our secondary purpose is to initiate the explicit study of CCPs by consecutive (or ridge) patterns. Our introduction of two-column ridge patterns provides a unifying characterization of the common subclasses of CCPs. In subsections 7.1 and 7.2, we use results on words to enumerate directed CCPs by two-column ridge patterns and by valleys. The Temperley method as modified in [4] is employed in subsection 9.3 to count CCPs by peaks.

We begin our exposé of (1) with a discussion of $\mathcal{P} \mathcal{S}$ and then work our way up the sequence of inclusions.

\section{Consecutive patterns in permutations}

Let $S_{n}$ denote the set of permutations of $1,2, \ldots, n$. When a permutation $\sigma=\sigma_{1} \sigma_{2} \ldots \sigma_{n} \in$ $S_{n}$ is sketched in a natural way, patterns take shape. In the sketch of $\sigma=256143 \in S_{6}$ in Diagram 1, one discerns ascents, descents, peaks, valleys, and other patterns. For $\sigma \in S_{n}$ and $p \in S_{m}$ with $m \leqslant n$, a segment $s=\sigma_{k} \sigma_{k+1} \ldots \sigma_{k+m-1}$ in $\sigma$ is referred to as a consecutive $p$-pattern if the relative order of the integers in $s$ agrees with the relative order of the integers in $p$ (that is, $\sigma_{k+j-1}$ is the $p_{j}^{t h}$ smallest integer in the list $\sigma_{k}, \sigma_{k+1}, \ldots, \sigma_{k+m-1}$ ).

\section{Diagram 1}

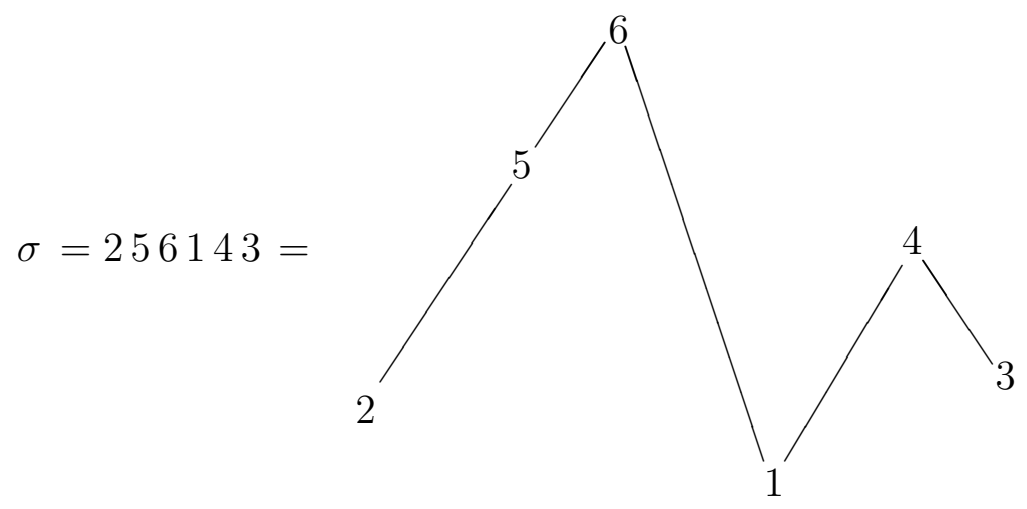

In Diagram 1, the ascent $\sigma_{2} \sigma_{3}=56$ is a 12 -pattern and the segment $\sigma_{2} \sigma_{3} \sigma_{4}=561$ is a 231-pattern. A segment that is either a 132-pattern or a 231-pattern is a peak. A peak is therefore a set of patterns. 
There are two standard ways of counting the number of times a given set of patterns $P \subseteq \bigcup_{m \geqslant 1} S_{m}$ occurs consecutively in a permutation $\sigma \in S_{n}$ :

- $P(\sigma)=$ the total number of times elements of $P$ occur in $\sigma$ and

- $P_{n o}(\sigma)=$ the maximum number of non-overlapping times elements of $P$ occur in $\sigma$.

When $P$ is of cardinality 1 , say $P=\{p\}$, we write $p(\sigma)$ and $p_{n o}(\sigma)$ in place of $P(\sigma)$ and $P_{n o}(\sigma)$. Relative to Diagram $1,132(\sigma)=1=132_{n o}(\sigma)$. For pic $=\{132,231\}$, note that $\operatorname{pic}(\sigma)=2$ whereas $\operatorname{pic}_{n o}(\sigma)=1$ (since the peaks $\sigma_{2} \sigma_{3} \sigma_{4}=561$ and $\sigma_{4} \sigma_{5} \sigma_{6}=143$ overlap at $\sigma_{4}=1$ ).

For a pattern set $P \subseteq \bigcup_{m \geqslant 1} S_{m}$, two primary enumeration questions arise:

- Q1: What is the cardinality of $P S_{n}=P \cap S_{n}$ ? Elements of $P S_{n}$ are referred to as $P$-pattern permutations of length $n$.

- Q2: How many permutations in $S_{n}$ contain $k$ consecutive $P$-patterns, counting overlaps?

The variation of Q2 involving the maximal number of non-overlapping patterns will be denoted by $\mathrm{Q} 2_{n o}$. The problem of counting permutations that contain no $P$-patterns is known as the avoidance problem. The pattern avoidance cases $(k=0)$ of Q2 and Q2 ${ }_{n o}$ are identical as $\left\{\sigma \in S_{n}: P(\sigma)=0\right\}=\left\{\sigma \in S_{n}: P_{n o}(\sigma)=0\right\}$.

As will be seen, there is a hierarchy between some versions of Q1, Q2, and Q2 $2_{n o}$; in these cases, solving Q1 solves Q2, which in turn solves Q2 ${ }_{n o}$. Our placement of the problem Q1 of enumerating permutations replete with $P$-patterns at the top of the hierarchy complements and sharply contrasts with the central role played in [23, 29] of the avoidance problem of counting permutations devoid of $P$ in solving $\mathrm{Q} 22_{n o}$.

\subsection{Selected examples}

In 1881, André [1] solved what has become the classic example of Q1. For UD $=\bigcup_{m \geqslant 1}\{p \in$ $\left.S_{m}: p_{1}<p_{2}>p_{3}<p_{4}>\cdots\right\}$, the elements of UD $S_{n}$ are said to be up-down permutations of length $n$. André showed that

$$
\sum_{n \geqslant 0}\left|\operatorname{UD} S_{n}\right| \frac{z^{n}}{n !}=\sec z+\tan z .
$$

As an example of Q2, we present the generating function for permutations by peaks obtained by Mendes and Remmel [29]:

$$
\sum_{n \geqslant 0} \sum_{\sigma \in S_{n}} \frac{y^{\mathrm{pic}(\sigma)} z^{n}}{n !}=\frac{\sqrt{y-1}}{\sqrt{y-1}-\tan (z \sqrt{y-1})} .
$$

Prior to [29], Kitaev [25] obtained a different form for the right side of (3). Incidentally, Entringer [13] enumerated "circular" permutations by peaks. 
The appearance of the tangent function in both (2) and (3) is no coincidence. A general explanation is provided in section 5, thereby showing that solving Q1 solves Q2.

The $q$-shifted factorial of an integer $n \geqslant 0$ is $(t ; q)_{n}=\prod_{k=0}^{n-1}\left(1-t q^{k}\right)$. The inversion number of a permutation $\sigma \in S_{n}$, defined by

$$
\operatorname{inv} \sigma=\mid\left\{(i, j): 1 \leqslant i<j \leqslant n \text { and } \sigma_{i}>\sigma_{j}\right\} \mid
$$

gives rise to many natural $q$-analogs. For instance, Gessel [16] and Mendes and Remmel [29] respectively showed that

$$
\begin{aligned}
\sum_{n \geqslant 0} \sum_{\sigma \in \mathrm{UD} S_{n}} \frac{q^{\operatorname{inv} \sigma} z^{n}}{(q ; q)_{n}} & =\sec _{q} z+\tan _{q} z \quad \text { and } \\
\sum_{n \geqslant 0} \sum_{\sigma \in S_{n}} \frac{y^{\operatorname{pic}(\sigma)} q^{\operatorname{inv} \sigma} z^{n}}{(q ; q)_{n}} & =\frac{\sqrt{y-1}}{\sqrt{y-1}-\tan _{q}(z \sqrt{y-1})}
\end{aligned}
$$

with $\cos _{q} z=\sum_{n \geqslant 0}(-1)^{n} z^{2 n} /(q ; q)_{2 n}, \sin _{q} z=\sum_{n \geqslant 0}(-1)^{n} z^{2 n+1} /(q ; q)_{2 n+1}, \sec _{q} z=1 / \cos _{q} z$, and $\tan _{q} z=\sin _{q} z / \cos _{q} z$. Replacing $z$ by $z(1-q)$ and then letting $q$ approach 1 reduces (4) to (2); hence (4) is a $q$-analog of (2). Likewise, (5) is a $q$-analog of (3).

\subsection{Solving $\mathrm{Q} 2$ solves $\mathrm{Q} 2_{n o}$}

In [23], Kitaev made the beautiful observation that $\mathrm{Q} 2{ }_{\text {no }}$ for a single pattern may be reduced to the avoidance problem. Shortly thereafter, Mendes and Remmel [29] extended Kitaev's result by tracking a set of patterns and adding the inversion number to the mix.

Theorem 1 (Mendes and Remmel 2007). If $P \subseteq S_{m}$ with $m>1$, then

$$
\sum_{n \geqslant 0} \sum_{\sigma \in S_{n}} \frac{q^{\text {inv } \sigma} y^{P_{n o}(\sigma)} z^{n}}{(q ; q)_{n}}=\frac{\mathcal{K}_{q}(z)}{1-y+y\left(1-z(1-q)^{-1}\right) \mathcal{K}_{q}(z)}
$$

where $\mathcal{K}_{q}(z)=\sum_{n \geqslant 0}\left(\sum_{\sigma \in S_{n}} q^{\operatorname{inv} \sigma} 0^{P(\sigma)}\right) z^{n} /(q ; q)_{n}$ is the q-exponential generating function for permutations that avoid $P$.

Among many consequences of Theorem 1, Mendes and Remmel obtained a solution to $\mathrm{Q} 2{ }_{n o}$ relative to peaks:

$$
\sum_{n \geqslant 0} \sum_{\sigma \in S_{n}} \frac{q^{\mathrm{inv} \sigma} y^{\mathrm{pic}_{n o}(\sigma)} z^{n}}{(q ; q)_{n}}=\left(1-\frac{y z}{1-q}+\sqrt{-1}(1-y) \tan _{q}(z \sqrt{-1})\right)^{-1}
$$

Theorem 1 provides a bridge from some versions of Q2 to Q2 $2_{n o}$. For instance, setting $y=0$ in (5) gives the $q$-exponential generating function for peak-avoiding permutations, which in turn may be plugged into Theorem 1 to get (6). For this reason, our primary focus will be on Q2. 


\section{Consecutive patterns in compositions}

Let $K_{n}=\left\{w=w_{1} w_{2} \ldots w_{n}: w_{1}, w_{2}, \ldots, w_{n}\right.$ are positive integers $\}$. For $w \in K_{n}$, set $\operatorname{sum} w=w_{1}+w_{2}+\cdots+w_{n}$. An element $w \in K_{n}$ for which $\operatorname{sum} w=m$ is said to be a composition of $m$ into $n$ parts.

As with permutations, a sketch of a composition $w \in K_{n}$ reveals patterns. When $w=377254 \in K_{6}$ is sketched as in Diagram 2, one observes ascents, levels, descents, peaks, valleys, and more.

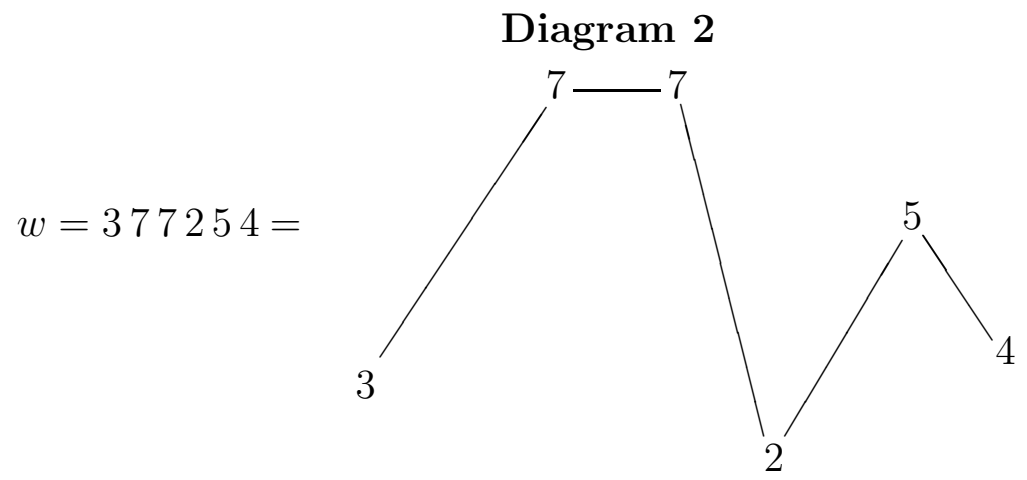

In particular, we define a peak in a composition $w$ to be a segment $w_{i} w_{i+1} w_{i+2}$ satisfying $w_{i} \leqslant w_{i+1}>w_{i+2}$. The number of peaks in $w$ is denoted by $\operatorname{pic}(w)$. In Diagram 2, segment $w_{2} w_{3} w_{4}=772$ is a peak and $\operatorname{pic}(w)=2$.

\subsection{Two revealing examples}

Naturally, Q1 and Q2 have been considered in the context of compositions. Paralleling André [1], a composition $w$ for which $w_{1} \leqslant w_{2}>w_{3} \leqslant w_{4}>\cdots$ is said to be up-down. If $\mathrm{UD} K_{n}$ denotes the set of up-down compositions of length $n$, then

$$
\sum_{n \geqslant 0} \sum_{w \in \mathrm{UD} K_{n}} q^{\operatorname{sum} w}(z / q)^{n}=\sec _{q} z+\tan _{q} z
$$

Carlitz [7] obtained a related result; he used $w_{1} \leqslant w_{2} \geqslant w_{3} \leqslant w_{4} \geqslant \cdots$ as the defining property of an up-down composition.

As an example of Q2 for compositions, the generating function for compositions by peaks (see section 5 for a proof) is

$$
\sum_{n \geqslant 0} \sum_{w \in K_{n}} y^{\mathrm{pic}(w)} q^{\mathrm{sum} w}(z / q)^{n}=\frac{\sqrt{y-1}}{\sqrt{y-1}-\tan _{q}(z \sqrt{y-1})} .
$$

Heubach and Mansour [20] obtained the distributions for compositions with parts in an arbitrary alphabet by various three-letter patterns; their result for peaks is more general than (8).

Comparison of (4) with (7) and of (5) with (8) strongly suggests that certain problems in $\mathcal{P S}$ and $\mathcal{P C}$ are one-in-the-same. Fédou's [15] insertion-shift bijection provides the connection. 


\section{$3.2 \quad$ Fédou's bijection: $\mathcal{P S} \subset \mathcal{P C}$}

For $\sigma \in S_{n}$ and $1 \leqslant i \leqslant n$, set $\operatorname{inv}_{i} \sigma=\left|\left\{k: i<k \leqslant n, \sigma_{i}>\sigma_{k}\right\}\right|$. Also, let $\Lambda_{n}=\{w \in$ $\left.K_{n}: w_{1} \leqslant w_{2} \leqslant \cdots \leqslant w_{n}\right\}$. The inverse of Fédou's [15] insertion-shift bijection $\nabla_{n}$ : $S_{n} \times \Lambda_{n} \rightarrow K_{n}$, as personally communicated by Foata, is given by the rule $\nabla_{n}(\sigma, \lambda)=w$ where $w_{i}=\operatorname{inv}_{i} \sigma+\lambda_{\sigma_{i}}$. For example,

$$
\nabla_{6}(256143,224444)=377254 \text {. }
$$

There are two key properties to note. First, if $\nabla_{n}(\sigma, \lambda)=w$, then

$$
\operatorname{inv} \sigma+\operatorname{sum} \lambda=\operatorname{sum} w .
$$

Second, $\nabla_{n}$ roughly transfers the overall shape and patterns of $\sigma$ to the corresponding $w$. Relative to (9), $\sigma=256143 \in S_{6}$ and $w=377254 \in K_{6}$ are of similar shape (see Diagrams 1 and 2). Moreover, the peaks 561 and 143 in $\sigma=256143$ coincide with the peaks 772 and 254 in $w=377254$. The explanation behind $\nabla_{n}$ 's preservation of overall shape lies in the fact that, if $\nabla_{n}(\sigma, \lambda)=w$ and $1 \leqslant i<m \leqslant n$, then

$$
\sigma_{i}<\sigma_{m} \text { if and only if } w_{i} \leqslant w_{m}+\left|\left\{j: i<j<m, \sigma_{i}>\sigma_{j}\right\}\right| .
$$

In particular, $\nabla_{n}$ preserves peaks: (11) implies that $\sigma_{k}<\sigma_{k+1}>\sigma_{k+2}$ if and only if $w_{k} \leqslant w_{k+1}>w_{k+2}$.

Rather than defining consecutive patterns directly on compositions, it is convenient to take an indirect path through $\nabla_{n}$. For $p \in S_{m}$, the segment $w_{k} w_{k+1} \ldots w_{k+m-1}$ is said to be a consecutive $p$-pattern in $w$ provided the corresponding segment $\sigma_{k} \sigma_{k+1} \ldots \sigma_{k+m-1}$ is a consecutive $p$-pattern in the unique permutation $\sigma$ satisfying $w=\nabla_{n}(\sigma, \lambda)$. Furthermore, for $P \subseteq \cup_{m \geqslant 1} S_{m}$ and $w=\nabla_{n}(\sigma, \lambda)$, we define $P(w)=P(\sigma)$ and $P_{n o}(w)=P_{n o}(\sigma)$.

The definition of patterns for compositions through $\nabla_{n}$ has at least one shortcoming. For instance, $w_{k} w_{k+1}$ is a 12-pattern in $w$ if $w_{k} \leqslant w_{k+1}$. From the perspective of compositions though, distinguishing between the case $w_{k}<w_{k+1}$ and the case $w_{k}=w_{k+1}$ may well be of interest. So there are problems in $\mathcal{P C}$ that have no analog in $\mathcal{P} \mathcal{S}$. However, $\mathcal{P S} \subset \mathcal{P C}$.

Theorem 2. If $P \subseteq \bigcup_{m \geqslant 1} S_{m}$ and if $B_{n} \subseteq S_{n}$, then

$$
\sum_{n \geqslant 0} \sum_{\sigma \in B_{n}}\left(\prod_{p \in P} y_{p}^{p(\sigma)}\right) \frac{q^{\mathrm{inv} \sigma} z^{n}}{(q ; q)_{n}}=\sum_{n \geqslant 0} \sum_{w \in \nabla_{n}\left(B_{n}, \Lambda_{n}\right)}\left(\prod_{p \in P} y_{p}^{p(w)}\right) q^{\mathrm{sum} w}(z / q)^{n} .
$$

Moreover, the above equality remains true if $y_{p}^{p(\sigma)}$ and $y_{p}^{p(w)}$ are respectively replaced by $y_{p}^{p_{n o}(\sigma)}$ and $y_{p}^{p_{n o}(w)}$ for some or all $p \in P$.

Proof. We prove the first assertion. As is well known, $(q ; q)_{n}^{-1}=\sum_{\lambda \in \Lambda_{n}} q^{\operatorname{sum} \lambda-n}$. By the properties of $\nabla_{n}$,

$$
\begin{aligned}
\sum_{n \geqslant 0} \sum_{\sigma \in B_{n}}\left(\prod_{p \in P} y_{p}^{p(\sigma)}\right) \frac{q^{\mathrm{inv} \sigma} z^{n}}{(q ; q)_{n}} & =\sum_{n \geqslant 0} \sum_{\sigma \in B_{n}} \sum_{\lambda \in \Lambda_{n}}\left(\prod_{p \in P} y_{p}^{p(\sigma)}\right) q^{\operatorname{inv} \sigma+\operatorname{sum} \lambda}(z / q)^{n} \\
& =\sum_{n \geqslant 0} \sum_{w \in \nabla_{n}\left(B_{n}, \Lambda_{n}\right)}\left(\prod_{p \in P} y_{p}^{p(w)}\right) q^{\operatorname{sum} w}(z / q)^{n} .
\end{aligned}
$$


There are three immediate applications of Theorem 2. First, Theorem 2 may be used to deduce (8) from Mendes and Remmel's (5). Likewise, (7) follows from Gessel's (4). Finally, Theorem 2 may be used to rewrite Mendes and Remmel's Theorem 1 in the context of compositions.

Corollary 1. If $P \subseteq S_{m}$ with $m>1$, then

$$
\sum_{n \geqslant 0} \sum_{w \in K_{n}} y^{P_{n o}(w)} q^{\operatorname{sum} w} z^{n}=\frac{L_{q}(z)}{1-y+y\left(1-z q(1-q)^{-1}\right) L_{q}(z)}
$$

where $L_{q}(z)=\sum_{n \geqslant 0}\left(\sum_{w \in K_{n}} q^{\mathrm{sum} w} 0^{P(w)}\right) z^{n}$ is the generating function for compositions that avoid $P$.

Corollary 1 is both more and less general than Heubach, Kitaev, and Mansour's [22] Theorem 4.1; for a pattern set of cardinality 1, their result holds for an arbitrary alphabet of positive integers.

\subsection{Compositions by two-term patterns and variation}

In a composition $w$, a segment $w_{k} w_{k+1}$ is said to be an ascent, level, or descent respectively as $w_{k}<w_{k+1}, w_{k}=w_{k+1}$, or $w_{k}>w_{k+1}$. The numbers of ascents, levels, and descents in $w$ are denoted by asc $w$, lev $w$, and des $w$. When sketched as in Diagram 2, one of the more compelling features of a composition $w \in K_{n}$ is its vertical variation defined by

$$
\operatorname{var} w=\sum_{k=0}^{n}\left|w_{k+1}-w_{k}\right| \quad \text { where, by convention, } w_{0}=0=w_{n+1} \text {. }
$$

As a consequence of the perspective afforded by (1), we obtain the following joint distribution of (asc, lev, des, var) on compositions from our Corollary 7 on directed columnconvex polyominoes recorded in subsection 7.1 .

Corollary 2. The generating function for compositions by ascents, levels, descents, and variation $K(c, z)=\sum_{n \geqslant 0} \sum_{w \in K_{n}} a^{\text {asc } w} b^{\operatorname{lev} w} d^{\operatorname{des} w} c^{\operatorname{var} w} q^{\operatorname{sum} w} z^{n}$ is given by

$$
K(c, z)=1+\frac{c^{2} \sum_{n \geqslant 0} \frac{(q z)^{n+1}}{1-c^{2} q^{n+1}} \prod_{k=1}^{n}\left(b+\frac{c^{2} d q^{k}}{1-c^{2} q^{k}}-\frac{a}{1-q^{k}}\right)}{1-a \sum_{n \geqslant 1} \frac{(q z)^{n}}{1-q^{n}} \prod_{k=1}^{n-1}\left(b+\frac{c^{2} d q^{k}}{1-c^{2} q^{k}}-\frac{a}{1-q^{k}}\right)} .
$$

Setting $c=1$ in Corollary 2 and making use of Cauchy's $q$-binomial theorem gives Carlitz's [6] generating function $K(1, z)$ for compositions by ascents, levels, and descents. Heubach and Mansour [21] recently extended Carlitz's result to an arbitrary alphabet of positive integers. 
The distributions of var and of closely related statistics over various combinatorial sets have been considered in $[2,28,33,38]$. In [38], Tiefenbruck expressed the generating function for compositions with bounded parts by variation as a ratio of coefficients of basic hypergeometric series. Recently, Mansour [28] determined the generating function for the same version of var on compositions as in [2].

\section{Factors and consecutive patterns in words}

Let $X^{*}$ be the free monoid generated by a nonempty alphabet $X$. The number of letters in a word $w \in X^{*}$ is referred to as its length and is denoted by len $w$. Set $X^{n}=\{w \in$ $\left.X^{*}: \operatorname{len} w=n\right\}$ and $X^{+}=\left\{w \in X^{*}:\right.$ len $\left.w>0\right\}$. The $k$ th letter of a word $w$ will be denoted by $w_{k}$; so $w=w_{1} w_{2} \ldots w_{\text {len } w}$.

An element $f \in X^{+}$is a factor of $w \in X^{*}$ if $f=w_{k} w_{k+1} \ldots w_{k+\operatorname{len} f-1}$ for some $k$. The number of times $f$ appears as a factor in $w$ is denoted by $f(w)$.

For a nonempty set $\mathcal{F} \subseteq X^{+}$, a factor $f$ of a word $w$ is a said to be a consecutive $\mathcal{F}$-pattern in $w$ if $f \in \mathcal{F}$. The number of consecutive $\mathcal{F}$-patterns in $w$ is denoted by $\mathcal{F}(w)$; so $\mathcal{F}(w)=\sum_{f \in \mathcal{F}} f(w)$. We refer to $\mathcal{F}$ as a factor set.

The containment $\mathcal{P C} \subset \mathcal{P W}$ in (1) is now evident: A composition $w$ is just a word with letters selected from the alphabet $N=\{1,2,3, \ldots\}$. In fact, $N^{*}=\cup_{n \geqslant 0} K_{n}$. Also, each pattern $p$ of length $m$ defined on compositions may be naturally matched with the factor set $\mathcal{F}_{p}=\left\{f \in N^{m}: p(f)=1\right\}$. For $p=132$ defined on compositions through Fédou's bijection as in subsection 3.2, $\mathcal{F}_{132}=\left\{a c b \in N^{3}: a \leqslant b<c\right\}$. In general, for a pattern set $P$ on compositions, we define $\mathcal{F}_{P}=\cup_{p \in P} \mathcal{F}_{p}$ and note that $P(w)=\mathcal{F}_{P}(w)$.

As a result, any method for the set $\mathcal{P W}$ may be applied to the set $\mathcal{P C}$ and, via Theorem 2, to $\mathcal{P S}$. In this regard, some modifications of Goulden and Jackson's [17] result for enumerating words by factors are fundamental.

As in Stanley [36, p. 266-267], we state Goulden and Jackson's [17] result in the context of the free monoid. Following Noonan and Zeilberger [31], the stipulation that no element of the factor set $\mathcal{F}$ be a factor of another is dropped. We further drop the requirement that the alphabet be finite, and we consider restrictions on the first and last letters.

For a nonempty set $\mathcal{F} \subset X^{+}$, an $\mathcal{F}$-cluster is a triple $(w, \nu, \beta)$ in which

$$
\begin{aligned}
& w=w_{1} w_{2} \ldots w_{\text {len } w} \in X^{+}, \\
& \nu=\left(f_{(1)}, f_{(2)}, \ldots, f_{(k)}\right) \text { for some } k \geqslant 1 \text { with each } f_{(i)} \in \mathcal{F}, \text { and } \\
& \beta=\left(b_{1}, b_{2}, \ldots, b_{k}\right) \text { with each } b_{i} \text { being a positive integer }
\end{aligned}
$$

where $f_{(i)}=w_{b_{i}} w_{b_{i}+1} \ldots w_{b_{i}+\operatorname{len} f_{(i)}-1}$, each $w_{i} w_{i+1}$ is a factor of some $f_{(j)}, b_{1} \leqslant b_{2} \leqslant \cdots \leqslant b_{k}$, and if $b_{i}=b_{i+1}$, then len $f_{(i)}<\operatorname{len} f_{(i+1)}$.

Roughly speaking, the pair $(\nu, \beta)$ is a recipe for covering $w$ with $\mathcal{F}$-factors: $\beta$ specifies where the factors in $\nu$ are to be "placed so as to cover" $w$. Accordingly, $w$ is said to be $\mathcal{F}$-coverable and the pair $(\nu, \beta)$ is said to be a covering of $w$. We let $C_{\mathcal{F}}$ denote the set of $\mathcal{F}$-clusters. 
For nonempty $A, B \subseteq X^{*}$, define $A B=\{a b: a \in A, b \in B\}$. The cluster generating function over a subset $W$ of $X^{*}$ is defined to be the formal series

$$
C_{\mathcal{F}}(\mathbf{y}, W)=\sum_{\substack{(w, \nu, \beta) \in C_{\mathcal{F}} \\ w \in W}}\left(\prod_{f \in \mathcal{F}} y_{f}^{f(\nu)}\right) w
$$

where $f(\nu)$ is the number of times $f$ appears as a component in $\nu$. With but trivial changes, Stanley's solution to problem 14(a) in [36, p. 266-267] establishes the following theorem.

Theorem 3 (Modifications of Goulden and Jackson's [17] result). If, for nonempty $L, R \subseteq$ $X$ and a nonempty $\mathcal{F} \subseteq X^{+}$, we define

$$
\begin{aligned}
& \mathcal{L}(\mathbf{y})=\sum_{l \in L} l+C_{\mathcal{F}}\left(\mathbf{y}, L X^{*}\right), \quad \mathcal{R}(\mathbf{y})=\sum_{r \in R} r+C_{\mathcal{F}}\left(\mathbf{y}, X^{*} R\right), \text { and } \\
& \mathcal{X}(\mathbf{y})=\sum_{x \in X} x+C_{\mathcal{F}}\left(\mathbf{y}, X^{*}\right)
\end{aligned}
$$

and if the result of replacing each $y_{f}$ in $\mathbf{y}$ by $y_{f}-1$ is denoted by $\mathbf{y}-\mathbf{1}$, then

$$
\begin{aligned}
\sum_{w \in X^{*}}\left(\prod_{f \in \mathcal{F}} y_{f}^{f(w)}\right) w & =(1-\mathcal{X}(\mathbf{y}-\mathbf{1}))^{-1}, \\
\sum_{w \in L X^{*}}\left(\prod_{f \in \mathcal{F}} y_{f}^{f(w)}\right) w & =\mathcal{L}(\mathbf{y}-\mathbf{1})(1-\mathcal{X}(\mathbf{y}-\mathbf{1}))^{-1}, \\
\sum_{w \in X^{*} R}\left(\prod_{f \in \mathcal{F}} y_{f}^{f(w)}\right) w & =(1-\mathcal{X}(\mathbf{y}-\mathbf{1}))^{-1} \mathcal{R}(\mathbf{y}-\mathbf{1}), \text { and } \\
\sum_{w \in L X^{*} R}\left(\prod_{f \in \mathcal{F}} y_{f}^{f(w)}\right) w & =C_{\mathcal{F}}\left(\mathbf{y}-\mathbf{1}, L X^{*} R\right)+\mathcal{L}(\mathbf{y}-\mathbf{1})(1-\mathcal{X}(\mathbf{y}-\mathbf{1}))^{-1} \mathcal{R}(\mathbf{y}-\mathbf{1}) .
\end{aligned}
$$

\section{$5 \quad$ Application of Theorem 3 to $\mathcal{P S}$ (and $\mathcal{P C}$ )}

In light of subsection 2.2 (solving Q2 solves Q2 ${ }_{n o}$ ), we focus on Q2. We begin with a useful digression into the setting of compositions.

Consider the alphabet $N=\{1,2,3, \ldots\}$, let $P \subseteq \bigcup_{m \geqslant 1} S_{m}$, and put

$$
D_{P}(\mathbf{y} ; z)=\sum_{(w, \nu, \beta) \in C_{\mathcal{F}_{P}}}\left(\prod_{p \in P} y_{p}^{p(\nu)}\right) q^{\operatorname{sum} w} z^{\operatorname{len} w} \text { where } p(\nu)=\sum_{f \in \mathcal{F}_{p}} f(\nu) .
$$

Replacement of $w$ by $q^{\operatorname{sum} w} z^{\operatorname{len} w}$ in the first identity of Theorem 3 yields

$$
\sum_{n \geqslant 0} \sum_{w \in K_{n}}\left(\prod_{p \in P} y_{p}^{p(\nu)}\right) q^{\mathrm{sum} w} z^{n}=\left(1-z q(1-q)^{-1}-D_{P}(\mathbf{y}-\mathbf{1} ; z)\right)^{-1} .
$$


Besides being a practical tool for enumerating compositions by patterns, (12) also reveals the fact that solving Q1 solves Q2 for compositions. To illustrate both points, we deduce (8) from (7) and (12). Relative to pic $=\{132,231\}$, set $y_{132}=y_{231}=y$. As the picclusters are in one-to-one correspondence with the up-down compositions of odd length greater than 1 ,

$$
\frac{z}{1-q}+D_{P}(y ; z / q)=\frac{1}{\sqrt{y}} \sum_{n \geqslant 0} \sum_{w \in \mathrm{UDK} K_{2 n+1}} q^{\operatorname{sum} w}(z \sqrt{y} / q)^{2 n+1} .
$$

So, (12) with $z$ replaced by $z / q$ and the odd part of (7) imply (8). Thus, counting up-down compositions solves the problem of counting compositions by peaks.

Theorem 2 allows the considerations of the above paragraph to be rephrased in the context of permutations. So, for permutations, solving Q1 solves Q2. Also, Theorem 2 applied to the lefthand side of (12) implies Theorem 4.

Theorem 4. If $P \subseteq \bigcup_{m \geqslant 1} S_{m}$, then

$$
\sum_{n \geqslant 0} \sum_{\sigma \in S_{n}}\left(\prod_{p \in P} y_{p}^{p(\sigma)}\right) \frac{q^{\mathrm{inv} \sigma} z^{n}}{(q ; q)_{n}}=\left(1-z(1-q)^{-1}-D_{P}(\mathbf{y}-\mathbf{1} ; z / q)\right)^{-1}
$$

Theorem 4 strengthens the main result in Rawlings [34] by dropping the restriction that $P$ be permissible (that is, no $p \in P$ occurs as a consecutive pattern in another $r \in P$ ). The restricted result in [34] was used to extend some permutation results of Elizalde and Noy's [13] as well as to solve a few other problems in $\mathcal{P} \mathcal{S}$. The example of subsection 5.3 involves a non-permissible $P$.

For $P=\left\{p \in S_{m}: p_{1} *_{1} p_{2} *_{2} \cdots *_{m-1} p_{m}\right\}$ where $*_{1}, *_{2}, \ldots, *_{m-1} \in\{<,>\}$, there are two common types of problems in $\mathcal{P} \mathcal{S}$ to be considered. The first is to track $P$ as a whole and the second involves tracking the patterns in $P$ individually. Relative to $\mathrm{Q} 2$, these respective problems are to determine

$$
\sum_{\sigma \in S_{n}} y^{P(\sigma)} q^{\mathrm{inv} \sigma} \text { and } \sum_{\sigma \in S_{n}}\left(\prod_{p \in P} y_{p}^{p(\sigma)}\right) q^{\mathrm{inv} \sigma} .
$$

To illustrate the use of Theorem 4, we will apply it to deduce four new results. The examples in subsections 5.1 and 5.2 track particular pattern sets as wholes, the example of subsection 5.3 tracks two pattern sets of different lengths, and the example of subsection 5.4 tracks patterns individually. In doing these examples, we must enumerate permutations by up-down type.

\subsection{Permutations by (i,d)-peaks and by up-down type}

For $i, d \geqslant 2$, let $P_{i, d}=\left\{p \in S_{i+d-1}: p_{1}<p_{2}<\cdots<p_{i}>p_{i+1}>\cdots>p_{i+d-1}\right\}$. A consecutive occurrence of a $P_{i, d}$-pattern in a permutation $\sigma$ is said to be an $(i, d)$-peak. In Diagram $1, \sigma_{1} \sigma_{2} \sigma_{3} \sigma_{4}=2561$ is a $(3,2)$-peak. Of course, a $(2,2)$-peak is just a peak 
as defined in subsection 2.1. Theorems 3 and 4 may be used to obtain the generating function for permutations by $(i, d)$-peaks as rational expressions of $q$-Olivier functions

$$
\Phi_{i, k}(z)=\sum_{n \geqslant 0} \frac{z^{i n+k}}{(q ; q)_{i n+k}}
$$

To this end, for $i_{1}, d_{1}, \ldots, i_{m}, d_{m} \geqslant 2$ and $k \geqslant 1$, let $\mathrm{UD} K_{i_{1}, d_{1} ; \cdots ; i_{m}, d_{m} ; k}$ denote the set of compositions $w$ that begin with a weakly increasing sequence $w_{1} \leqslant w_{2} \leqslant \cdots \leqslant w_{i_{1}}$ of length $i_{1}$, then continue with a strictly decreasing sequence $w_{i_{1}}>w_{i_{1}+1}>\cdots>w_{i_{1}+d_{1}-1}$ of length $d_{1}$, followed by a weakly increasing sequence of length $i_{2}$, then a strictly decreasing sequence of length $d_{2}$, and so on until ending with a weakly increasing sequence of length $k$.

We let $(j, d)^{m}$ denote the list $j, d ; j, d ; \ldots ; j, d$ in which $j, d$ appears $m$ times. A composition in $\mathrm{UD} K_{i, d ;(j, d)^{m} ; k}$, for any $m \geqslant 0$, is said to be of up-down type $(i, j, d ; k)$. Up-down permutations of type $(i, j, d ; k)$ are similalry defined.

Corollary 3. If, for $i, j, d \geqslant 2$, we set $\mu=i+d-2$ and $\xi_{m}=\sqrt[m]{-1}$, then the generating function for permutations by $(i, d)$-peaks and inversions is

$$
\sum_{n \geqslant 0} \sum_{\sigma \in S_{n}} \frac{y^{P_{i, d}(\sigma)} q^{\mathrm{inv} \sigma} z^{n}}{(q ; q)_{n}}=\left(1-z(1-q)^{-1}-\frac{K_{i, i, d ; 1}(\sqrt[\mu]{y-1} z)}{\sqrt[\mu]{y-1}}\right)^{-1}
$$

where, for $k \geqslant 1$,

$$
K_{i, j, d ; k}(z)=\sum_{m \geqslant 0} \sum_{w \in K_{i, d ;(j, d)^{m} ; k}} q^{\text {sum } w} z^{\text {len } w} .
$$

Moreover, $K_{i, j, d ; k}(z)$ satisfies, for $d \geqslant 3$ and $\nu=j+d-2$, the recurrence

$$
\begin{array}{r}
K_{i, j, d ; k}(z)=\frac{\xi_{\nu}^{-\mu} K_{i, j+1, d-1 ; 1}\left(\xi_{\nu} z\right)\left(z^{k}(q ; q)_{k}^{-1}+\xi_{\nu}^{-k} K_{j, j+1, d-1 ; k+1}\left(\xi_{\nu} z\right)\right)}{1+K_{j, j+1, d-1 ; 1}\left(\xi_{\nu} z\right)} \\
-\xi_{\nu}^{-\mu-k} K_{i, j+1, d-1 ; k+1}\left(\xi_{\nu} z\right)
\end{array}
$$

with the initial condition $K_{i, j, 2 ; k}(z)=\xi_{j}^{-i-k}\left[\frac{\Phi_{j, i}\left(\xi_{j} z\right) \Phi_{j, k}\left(\xi_{j} z\right)}{\Phi_{j, 0}\left(\xi_{j} z\right)}-\Phi_{j, i+k}\left(\xi_{j} z\right)\right]$.

Before providing proof, a few examples are presented. First, the above recurrence provides a straightforward means of determining $K_{i, j, d ; k}(z)$ as a rational expression of $q$-Olivier functions. Therefore, the generating function for permutations by $(i, d)$-peaks given by Corollary 3 is also a rational expression in $q$-Olivier functions. For instance,

$$
\sum_{n \geqslant 0} \sum_{\sigma \in S_{n}} \frac{y^{P_{3,3}(\sigma)} q^{\mathrm{inv} \sigma} z^{n}}{(q ; q)_{n}}=\left(1-z(1-q)^{-1}-\frac{K_{3,3,3 ; 1}(\sqrt[4]{y-1} z)}{\sqrt[4]{y-1}}\right)^{-1}
$$

where

$$
K_{3,3,3 ; 1}(z)=\frac{-K_{3,4,2 ; 1}\left(\xi_{4} z\right)\left(z(1-q)^{-1}+\xi_{4}^{-1} K_{3,4,2 ; 2}\left(\xi_{4} z\right)\right)}{1+K_{3,4,2 ; 1}\left(\xi_{4} z\right)}+\xi_{4}^{-1} K_{3,4,2 ; 2}\left(\xi_{4} z\right)
$$


with

$$
\begin{aligned}
& K_{3,4,2 ; 1}(z)=-\frac{\Phi_{4,3}\left(\xi_{4} z\right) \Phi_{4,1}\left(\xi_{4} z\right)}{\Phi_{4,0}\left(\xi_{4} z\right)}+\Phi_{4,4}\left(\xi_{4} z\right) \text { and } \\
& K_{3,4,2 ; 2}(z)=\xi_{4}^{-1}\left[-\frac{\Phi_{4,3}\left(\xi_{4} z\right) \Phi_{4,2}\left(\xi_{4} z\right)}{\Phi_{4,0}\left(\xi_{4} z\right)}+\Phi_{4,5}\left(\xi_{4} z\right)\right] .
\end{aligned}
$$

Second, Corollary 3 and the comments of subsection 2.2 may be used to solve the Q2 $2_{n o}$ version of counting permutations by $(i, d)$-peaks. We illustrate by obtaining Mendes and Remmel's [29] result for the case $(i, 2)$. First, note that the initial condition at the end of Corollary 3 implies

$$
K_{i, i, 2 ; k}(z)=\frac{\xi_{i}^{-k} \Phi_{i, k}\left(\xi_{i} z\right)}{\Phi_{i, 0}\left(\xi_{i} z\right)}-\frac{z^{k}}{(q ; q)_{k}} .
$$

Substituting $K_{i, i, 2 ; 1}(z)$ into Corollary 3 , setting $y=0$, and plugging the result into Theorem 1 gives Mendes and Remmel's result, namely

$$
\sum_{n \geqslant 0} \sum_{\sigma \in S_{n}} \frac{y^{P_{i, 2 n o}(\sigma)} q^{\text {inv } \sigma} z^{n}}{(q ; q)_{n}}=\left(1-y z(1-q)^{-1}-\frac{(1-y) \Phi_{i, 1}(z)}{\Phi_{i, 0}(z)}\right)^{-1} .
$$

Third, by definition, $K_{i, j, d ; k}(z)$ is the generating function for up-down compositions of type $(i, j, d ; k)$. The classic result $z /(1-q)+K_{2,2,2 ; 1}(z)=\tan _{q} z$ for up-down compositions of odd length is evident in (13). Similarly, $z /(1-q)+K_{3,3,3 ; 1}(z)$ is the generating function for the so-called up-up-down-down compositions. Prodinger and Tshifhumulo [32] gave another recurrence, without obtaining a closed form, for the generating function for up-updown-down compositions. With " $\geqslant$ " in place of ">", Carlitz [7] determined the generating function for up-down compositions of type $(i, i, 2 ; k)$.

Finally, we again underscore the value of Theorem 2 in transcribing pattern results between the settings of compositions and permutations. For instance, replacing $z$ in the first part of Corollary 3 with $q z$ and invoking Theorem 2 gives the generating function for compositions by $(i, d)$-peaks.

Likewise, $K_{i, j, d ; k}(z / q)$ transcribes as the generating function for permutations by updown type $(i, j, d ; k)$ and by inversion number. So, $z /(1-q)+K_{3,3,3 ; 1}(z / q)$ is a $q$-analog of Carlitz and Scoville's [9] result for up-up-down-down permutations. Using another method, Mendes, Remmel, and Riehl [30] obtained generating functions for up-down permutations of type $(i, j, 2 ; k)$ with $k \leqslant j$. For up-down type $(0, j, 2 ; k)$, see Carlitz [5].

Proof of Corollary 3. The relevant cluster generating function is

$$
D_{P_{i, d}}(y ; z / q)=\sum_{(w, \nu, \beta) \in C_{\mathcal{F}_{P_{i, d}}}} q^{\mathrm{sum} w} y^{P_{i, d}(\nu)}(z / q)^{\text {len } w} .
$$


Clearly, a composition is $P_{i, d^{-}}$-coverable if and only if it belongs to $\mathrm{UD} K_{(i, d)^{m} ; 1}$ for some $m \geqslant 1$. Moreover, each $w \in U D K_{(i, d)^{m} ; 1}$ has but one $P_{i, d}$-covering. It follows that

$$
D_{P_{i, d}}(y ; z / q)=\frac{1}{\sqrt[\mu]{y}} \sum_{m \geqslant 1} \sum_{w \in \mathrm{UD} K_{(i, d)} m_{; 1}} q^{\operatorname{sum} w}(\sqrt[\mu]{y} z / q)^{\operatorname{len} w}=\frac{K_{i, i, d ; 1}(\sqrt[\mu]{y} z)}{\sqrt[\mu]{y}} .
$$

The above equality and Theorem 4 imply the first part of Corollary 3.

There are several theoretical frameworks (including the Pattern Algebra of Goulden and Jackson [18] described in section 8) for determining $K_{i, j, d ; k}(z)$. We will use Theorem 3 ; in this approach, up-down compositions having strictly descending runs of length $d$ are exchanged for "straighter" up-down compositions having strictly descending runs of length $d-1$.

Let $N_{i, d}=\left\{w \in N^{i+d-1}: w_{1} \leqslant w_{2} \leqslant \cdots \leqslant w_{i}>w_{i+1}>\cdots>w_{i+d-1}\right\}$. For any word $w$ in $N^{*}$ or in $N_{i, d}^{*}$, the symbol len $w$ is always to be interpreted as the length of $w$ relative to the alphabet $N$.

Relative to the alphabet $X_{d-1}=N_{k, 1} \bigcup\left(\bigcup_{l \geqslant 2} N_{l, d-1}\right)$, let $\mathcal{F}_{d-1}$ denote the set of words of the form $u v$ where $u, v \in X_{d-1}$ and such that the last letter in the factor $u$ is less than or equal to the first letter in $v$. For a word $w=u_{(1)} u_{(2)} \ldots u_{(n)}$ with each $u_{(m)} \in X_{d-1}$, let ris $w=\sum_{f \in \mathcal{F}_{d-1}} f(w)$.

As $\mathrm{UD} K_{i, d ;(j, d)^{m} ; k}=\left\{w \in N_{i, d-1} N_{j, d-1}^{*} N_{k, 1}:\right.$ ris $\left.w=0\right\}$, Theorem 3 leads to

$$
K_{i, j, d ; k}(z)=\sum_{m \geqslant 0} \sum_{w \in N_{i, d-1} N_{j, d-1}^{m} N_{k, 1}} 0^{\text {ris } w} q^{\operatorname{sum} w} z^{\operatorname{len} w}=A_{1, d}+\frac{A_{2, d} A_{3, d}}{1+A_{4, d}}
$$

where

$$
\begin{aligned}
& A_{1, d}=\sum_{m \geqslant 0}(-1)^{m+1} \sum_{\substack{N_{i, d-1} N_{j, d-1}^{m} N_{k, 1} \\
\operatorname{ris} w=m+1}} q^{\operatorname{sum} w} z^{\operatorname{len} w}, \quad A_{2, d}=\sum_{m \geqslant 0}(-1)^{m} \sum_{\substack{w \in N_{i, d-1} N_{j, d-1}^{m} \\
\operatorname{ris} w=m}} q^{\text {sum } w} z^{\operatorname{len} w},
\end{aligned}
$$

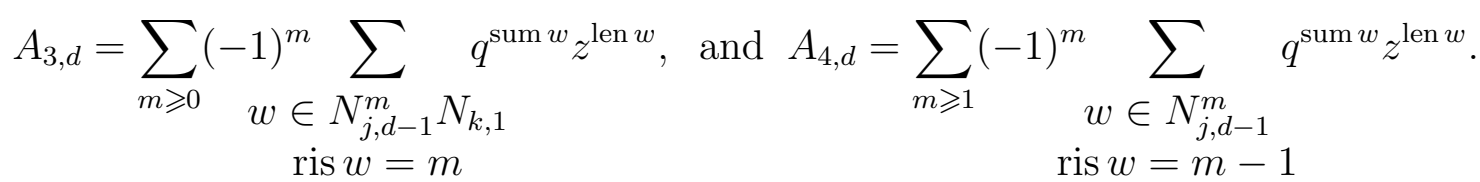

Completion of the proof is now just a matter of determining the sums $A_{l, d}$. Being of similar nature, only a few are evaluated here.

As an example of the case $d=2$, note that

$$
1+A_{4,2}=1+\sum_{m \geqslant 1}(-1)^{m} z^{j m} \sum_{0 \leqslant w_{1} \leqslant \cdots \leqslant w_{j m}} q^{w_{1}+\cdots+w_{j n}}=\sum_{m \geqslant 0} \frac{(-1)^{m} z^{j m}}{(q ; q)_{j m}}=\Phi_{j, 0}\left(\xi_{j} z\right) .
$$

For $A_{1, d}$ with $d \geqslant 3$, note that len $w=\mu+\nu m+k$ and that $\left\{w \in N_{i, d-1} N_{j, d-1}^{m} N_{k, 1}\right.$ : ris $w=m+1\}=\mathrm{UD} K_{i, d-1 ;(j+1, d-1)^{m} ; k+1}$. Thus, $A_{1, d}=-\xi_{\nu}^{-\mu-k} K_{i, j+1, d-1 ; k+1}\left(\xi_{\nu} z\right)$. 


\subsection{Uniform range distributions}

For $i, d \geqslant 2$ and $m \geqslant 1$, let $P_{(i, d)^{m}}$ denote the set of patterns $p \in S_{(i+d-2) m+1}$ that begin with an increasing sequence $p_{1}<\cdots<p_{i}$ of length $i$, continue with a decreasing sequence $p_{i}>p_{i+1}>\cdots>p_{i+d-1}$ of length $d$, followed by an increasing sequence $p_{i+d-1}<$ $p_{i+d}<\cdots<p_{2 i+d-2}$ of length $i$, and so on so as to form $m$ consecutive $(i, d)$-peaks. The consecutive occurrence of a $p \in P_{(i, d)^{m}}$ in a permutation $\sigma$ is said to be a uniform $m$-peak range of type $(i, d)$. The following result extends Corollary 3 to uniform ranges. The coefficient of $z^{n}$ in a formal power series $F(z)$ is denoted by $\left.F\right|_{z^{n}}$.

Corollary 4. If $i, d \geqslant 2, m \geqslant 1$, and $\nu=i+d-2$, then the generating function for permutations by uniform m-peak ranges and inversions is

$$
\sum_{n \geqslant 0} \sum_{\sigma \in S_{n}} \frac{y^{P_{(i, d)^{m}}(\sigma)} q^{\mathrm{inv} \sigma} z^{n}}{(q ; q)_{n}}=\left(1-\frac{z}{1-q}-\sum_{n \geqslant m} A_{n, m}(y-1) B_{n}(q) z^{n \nu+1}\right)^{-1}
$$

where

$$
A_{n, m}(y)=\left.\frac{y z^{m}(1-z)}{1-z-y z\left(1-z^{m}\right)}\right|_{z^{n}} \text { and } B_{n}(q)=\left.K_{i, i, d ; 1}(z)\right|_{z^{n \nu+1}}
$$

with $K_{i, i, d ; 1}(z)$ as determined in Corollary 3.

The case $i=d=2$ with $y=0$ of Corollary 4 provides a solution to the problem posed by Kitaev [25, Problem 1] of counting permutations that avoid $(2 m+1)$-reversealternating patterns (which, as noted in [25], is the same as the number of permutations that avoid $(2 m+1)$-alternating patterns). The case for even-length alternating patterns may be dealt with similarly.

Proof of Corollary 4. First, note that

$$
D_{P_{(i, d)^{m}}}(y ; z / q)=\sum_{(w, \nu, \beta) \in C_{\mathcal{F}}(i, d)^{m}} q^{\text {sum } w} y^{P_{(i, d)^{m}}(\nu)}(z / q)^{\operatorname{len} w} .
$$

Next, observe that a composition is $P_{(i, d)^{m}}$-coverable if and only if it belongs to the set of up-down compositions $\bigcup_{n \geqslant m} \mathrm{UD} K_{(i, d)^{n} ; 1}$. Moreover, there may be multiple $P_{(i, d)^{m}-}$ coverings $(\nu, \beta)$ for such a composition. For instance, $w=231423221 \in \mathrm{UD} K_{(2,2)^{4} ; 1}$ is

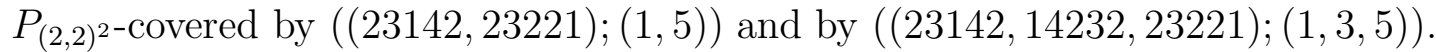

For $n \geqslant m \geqslant 1$ and $k \geqslant 1$, let $a_{n, m, k}$ denote the number of times that a given $w \in \mathrm{UD} K_{(i, d)^{n} ; 1}$ appears in a $P_{(i, d)^{m}}$-cluster $(w, \nu, \beta)$ with $P_{(i, d)^{m}}(\nu)=k$. Of course, $a_{n, m, k}$ is independent of the choice of $w \in \operatorname{UD} K_{(i, d)^{n} ; 1}$.

For $n \geqslant 1$, let

$$
A_{n, m}(y)=\sum_{k \geqslant 1} a_{n, m, k} y^{k} \text { and } B_{n}(q)=\sum_{w \in \mathrm{UD} K_{(i, d)} n_{i 1}} q^{\mathrm{sum} w} .
$$

Evidently, $B_{n}(q)=\left.K_{i, i, d ; 1}(z)\right|_{z^{n+1}}$. It follows that

$$
D_{P_{(i, d)^{m}}}(y ; z / q)=\sum_{n \geqslant m} A_{n, m}(y) B_{n}(q) z^{n \nu+1} .
$$


In view of Theorem 4 , we need only establish the formula for $A_{n, m}(y)$. Note that a typical $P_{(i, d)^{m}}$-cluster that contributes to the count $a_{n, m, k}$ is of the form $\left(w, \nu,\left(b_{1}, b_{2}, \ldots, b_{k}\right)\right)$ with $b_{2}$ equaling $i+d-1,2(i+d-2)+1, \ldots$, or $m(i+d-2)+1$. So, for $n \geqslant m \geqslant 1$ and $k \geqslant 2, a_{n, m, k}=\sum_{j=1}^{m} a_{n-j, m, k-1}$. Routine computations then lead to the fact that

$$
\sum_{n \geqslant m} \sum_{k \geqslant 1} a_{n, m, k} y^{k} z^{n}=\frac{y z^{m}(1-z)}{1-z-y z\left(1-z^{m}\right)} .
$$

Thus, $A_{n, m}(y)=\left.y z^{m}(1-z)\left(1-z-y z\left(1-z^{m}\right)\right)^{-1}\right|_{z^{n}}$.

\subsection{Permutations by peaks and twin peaks}

Let tpic $=\left\{p \in S_{5}: p_{1}<p_{2}>p_{3}<p_{4}>p_{5}\right\}$. A consecutive occurrence of $p \in$ tpic in a permutation is referred to as a twin peak. The set $P=$ pic $\cup$ tpic is not permissible and therefore not within the scope of the theorem in [34]. However, Theorem 4 makes the joint enumeration of permutations by peaks and twin peaks straightforward; we just need to determine

$$
D_{P}(x, y ; z / q)=\sum_{(w, \nu, \beta) \in C_{\mathcal{F}_{P}}} x^{\mathrm{pic}(\nu)} y^{\operatorname{tpic}(\nu)} q^{\operatorname{sum} w}(z / q)^{\text {len } w} .
$$

To this end, first note that the set of $P$-coverable compositions corresponds to the set of up-down compositions $\bigcup_{n \geqslant 1} \mathrm{UDK}_{2 n+1}$.

For $w \in \mathrm{UD} K_{2 n+1}$, let $a_{n, l, k}$ denote the number of $P$-coverings $(\nu, \beta)$ of $w$ by $l$ peaks and $k$ twin peaks. Set $A_{n}(x, y)=\sum_{l, k \geqslant 0} a_{n, l, k} x^{l} y^{k}$. From the easily deduced recurrence relationship

$$
a_{n, l, k}=a_{n-1, l-1, k}+a_{n-1, l-1, k-1}+a_{n-2, l-1, k-1}+a_{n-1, l, k-1}+a_{n-2, l, k-1},
$$

it follows that $A_{n}(x, y)=\left.\left(x z+y z^{2}+x y z^{2}\right)\left(1-x z-x y z-x y z^{2}-y z-y z^{2}\right)^{-1}\right|_{z^{n}}$.

Let $B_{n}(q)=\sum_{w \in \mathrm{UD} K_{2 n+1}} q^{\mathrm{sum} w}=\left.\tan _{q} z\right|_{z^{2 n+1}}$. In view of (14), we have

$$
D_{P}(x, y ; z / q)=\sum_{n \geqslant 1} A_{n}(x, y) B_{n}(q)(z / q)^{2 n+1}
$$

Finally, the last equality and Theorem 4 imply

$$
\sum_{n \geqslant 0} \sum_{\sigma \in S_{n}} \frac{q^{\mathrm{inv} \sigma} x^{\mathrm{pic}(\sigma)} y^{\mathrm{tpic}(\sigma)} z^{n}}{(q ; q)_{n}}=\left(1-\frac{z}{1-q}-\sum_{n \geqslant 1} A_{n}(x-1, y-1) B_{n}(q)(z / q)^{2 n+1}\right)^{-1} .
$$

Another solution to the joint peak and twin peak $\mathcal{P} \mathcal{S}$ is given in subsection 8.4. 


\subsection{Permutations and up-down permutations by (i,m)-maxima}

For $i \geqslant 2$ and $1 \leqslant m \leqslant i$, let $p_{(m)}$ denote the unique permutation in $S_{i+1}$ with $p_{(m) 1}<$ $p_{(m) 2}<\cdots<p_{(m) i}$ and $p_{(m) i+1}=i+1-m$. Also, let $P_{i}=\left\{p_{(1)}, p_{(2)}, \ldots, p_{(i)}\right\}$. A consecutive occurrence of $p_{(m)} \in P_{i}$ in a permutation $\sigma$ is said to be an $(i, m)$-maximum. Carlitz and Scoville [8] refer to (2,1)-maxima and (2,2)-maxima respectively as rising and falling maxima; they expressed the joint distribution of $\{12,132,231,21\}$ over $0 S_{n} 0=$ $\left\{0 \sigma 0: \sigma \in S_{n}\right\}$ in terms of a second order differential equation.

The statement of our next result requires the $q$-binomial coefficient which, for integers $n$ and $k$, is defined as

$$
\left[\begin{array}{l}
n \\
k
\end{array}\right]=\left\{\begin{array}{cl}
\left(q^{n-k+1} ; q\right)_{k} /(q ; q)_{k} & \text { if } n \geqslant k \geqslant 0 \\
0 & \text { otherwise. }
\end{array}\right.
$$

Corollary 5. If $i \geqslant 2$ and $1 \leqslant m \leqslant i$, then the generating function for permutations by (i,m)-maxima and inversions is

$$
\sum_{n \geqslant 0} \sum_{\sigma \in S_{n}}\left(\prod_{m=1}^{i} y_{m}^{p_{(m)}(\sigma)}\right) \frac{q^{\mathrm{inv} \sigma} z^{n}}{(q ; q)_{n}}=\left(1-\frac{\Phi_{i, 1}\left(\mathbf{y}-\mathbf{1} ; \xi_{i} z\right)}{\xi_{i} \Phi_{i, 0}\left(\mathbf{y}-\mathbf{1} ; \xi_{i} z\right)}\right)^{-1}
$$

where $\xi_{i}=\sqrt[i]{-1}$ and

$$
\Phi_{i, k}\left(y_{1}, \ldots, y_{i} ; z\right)=\sum_{n \geqslant 0} \frac{z^{i n+k}}{(q ; q)_{i n+k}} \prod_{j=0}^{n-1}\left(y_{i}+\sum_{m=1}^{i-1}\left(y_{i}-y_{m}\right) q^{m}\left[\begin{array}{c}
i j+k+m-1 \\
m
\end{array}\right]\right) .
$$

For $i=2, y_{1}=y$, and $y_{2}=1$, Corollary 5 gives the $q$-analog obtained in $[29,34]$ of Elizalde and Noy's [13] result for permutations by $p=132$ :

$$
\sum_{n \geqslant 0} \sum_{\sigma \in S_{n}} \frac{y^{132(\sigma)} q^{\mathrm{inv} \sigma} z^{n}}{(q ; q)_{n}}=\left(1-\sum_{n \geqslant 0} \frac{(y-1)^{n} q^{n} z^{2 n+1}}{\left(q^{2} ; q^{2}\right)_{n}\left(1-q^{2 n+1}\right)(1-q)^{n}}\right)^{-1} .
$$

Proof of Corollary 5. By Theorem 4, we need to compute

$$
D_{P_{i}}(\mathbf{y} ; z / q)=\sum_{(w, \nu, \beta) \in C_{\mathcal{F}}}\left(\prod_{m=1}^{i} y_{m}^{p_{(m)}(\nu)}\right) q^{\operatorname{sum} w}(z / q)^{\text {len } w} \quad \text { where } \mathcal{F}=\bigcup_{p \in P_{i}} \mathcal{F}_{p}
$$

Define $\mathcal{L}_{i}$ to be the set of compositions $w$ of length $i n+1$ for any $n \geqslant 0$ such that $w_{j}>w_{j+1}$ if and only if $j$ is a positive multiple of $i$. Note that a composition $w$ is $P_{i}$-coverable if and only if $w \in \mathcal{L}_{i}$ and len $w>1$. Moreover, such a composition has but one $P_{i}$-covering. Thus,

$$
\frac{z}{1-q}+D_{P_{i}}(\mathbf{y} ; z / q)=\sum_{w \in \mathcal{L}_{i}}\left(\prod_{p \in P_{i}} y_{p}^{p(w)}\right) q^{\operatorname{sum} w}(z / q)^{\operatorname{len} w}
$$


Let $\mathcal{L}_{i}\left(y_{1}, y_{2}, \ldots, y_{i} ; z\right)$ denote the right side of the above equality. For $w \in \mathcal{L}_{i}$ of length in +1 , observe that $p_{(1)}(w)+p_{(2)}(w)+\cdots+p_{(i)}(w)=n$. So

$$
\mathcal{L}_{i}\left(y_{1}, \ldots, y_{i} ; z\right)=y_{i}^{(-1 / i)} \mathcal{L}_{i}\left(y_{1} / y_{i}, \ldots, y_{i-1} / y_{i}, 1 ; z \sqrt[i]{y_{i}}\right) .
$$

We therefore only need to determine $\mathcal{L}_{i}\left(y_{1}, \ldots, y_{i-1}, 1 ; z\right)$.

Recall that $\Lambda_{i}=\left\{w \in N^{i}: w_{1} \leqslant w_{2} \leqslant \cdots \leqslant w_{i}\right\}$. As before, the symbol len $w$ denotes the length of $w$ relative to the alphabet $N$.

To determine $\mathcal{L}_{i}\left(y_{1}, \ldots, y_{i-1}, 1 ; z\right)$, we appeal to Theorem 3 . We work with the factor set $\mathcal{G}=\left\{u v: u \in \Lambda_{i}, v \in N\right\}$. For each $u v \in \mathcal{G}$, define

$$
y_{u v}=\left\{\begin{array}{cl}
t & \text { if } u_{i} \leqslant v \\
y_{m} & \text { if } u_{i+1-m}>v \geqslant u_{i-m} \text { for } 1 \leqslant m \leqslant i-1 \\
1 & \text { if } u_{1}>v
\end{array}\right.
$$

For $w=u_{(1)} u_{(2)} \ldots u_{(n)}$ with $u_{(1)}, \ldots, u_{(n-1)} \in \Lambda_{i}$ and $u_{(n)} \in N$, let ris $w$ be the number of indices $j$ such that the last letter of $u_{(j)}$ is less than or equal to the first letter of $u_{(j+1)}$. Note that $\prod_{g \in \mathcal{G}} y_{g}^{g(w)}=t^{\text {ris } w} \prod_{m=1}^{i-1} y_{m}^{p_{(m)}(w)}$. plies

As $\mathcal{L}_{i}\left(y_{1}, \ldots, y_{i-1}, 1 ; z\right)=\sum_{w \in \Lambda_{i}^{*} N} 0^{\text {ris } w} q^{\text {sum } w}(z / q)^{\text {len } w} \prod_{m=1}^{i-1} y_{m}^{p_{(m)}(w)}$, Theorem 3 im-

$$
\mathcal{L}_{i}\left(y_{1}, \ldots, y_{i-1}, 1 ; z\right)=\frac{\sum_{n \geqslant 0}(-1)^{n} z^{i n+1} \sum_{0 \leqslant \operatorname{sum} \alpha \leqslant n} \prod_{m=1}^{i-1}\left(1-y_{m}\right)^{\alpha_{m}} \sum_{w \in \mathcal{C}_{i, 1, n ; \alpha}} q^{\text {sum } w-i n-1}}{1+\sum_{n \geqslant 0}(-1)^{n} z^{i n+i} \sum_{0 \leqslant \operatorname{sum} \alpha \leqslant n} \prod_{m=1}^{i-1}\left(1-y_{m}\right)^{\alpha_{m}} \sum_{w \in \mathcal{C}_{i, i, n ; \alpha}} q^{\text {sum } w-i n-i}},
$$

where the sums on the right are over $\alpha \in\{0,1,2, \ldots\}\}^{i-1}$ and $w$ in $\mathcal{C}_{i, k, n ; \alpha}=\left\{w \in \Lambda_{i}^{n} \Lambda_{k}\right.$ : $p_{(i)}(w)=0$ and $p_{(m)}(w)=\alpha_{m}$ for $\left.1 \leqslant m \leqslant i-1\right\}$.

The proof is completed by showing that the numerator and denominator in (15) are respectively $\Phi_{i, 1}\left(y_{1}, \ldots, y_{i-1}, 1 ; \xi_{i} z\right) / \xi_{i}$ and $\Phi_{i, 0}\left(y_{1}, \ldots, y_{i-1}, 1 ; \xi_{i} z\right)$.

Another generating function for permutations by $(i, m)$-maxima is derived in subsection 9.2. Notably, Theorem 2 applied to the lefthand side of (15) yields the generating function for the set $\mathrm{UD} S_{i, i, 2 ; 1}$ of up-down permutations of type $(i, i, 2 ; 1)$ by $(i, m)$-maxima. Setting $y_{1}=y_{2}=\ldots=y_{i}=1$, replacing $z$ by $(1-q) z$, and letting $q \rightarrow 1$ in Corollary 6 gives a result of Carlitz's [5].

Corollary 6. For $i \geqslant 2$, the generating function for permutations of up-down type $(i, i, 2 ; 1)$ by $(i, m)$-maxima is given by

$$
\frac{z}{1-q}+\sum_{\sigma \in \mathrm{UD} S_{i, i, 2 ; 1}}\left(\prod_{m=1}^{i} y_{m}^{p_{(m)}}\right) \frac{q^{\mathrm{inv} \sigma} z^{\operatorname{len} \sigma}}{(q ; q)_{\operatorname{len} \sigma}}=\frac{\Phi_{i, 1}\left(y_{1}, \ldots, y_{i-1}, 1 ; \xi_{i} z\right)}{\xi_{i} \Phi_{i, 0}\left(y_{1}, \ldots, y_{i-1}, 1 ; \xi_{i} z\right)}
$$




\section{Ridge patterns in CCPs}

A column-convex polyomino is constructed by successively gluing a finite sequence of columns, each consisting of a finite number of unit square cells, together in the $x y$-plane so that (i) the lower left vertex of the leftmost column has coordinates $(0,0)$, (ii) each pair of adjacent columns share an edge of positive integer length, and (iii) all cell vertices have integer coordinates.

The area, perimeter, and number of columns of a column-convex polyomino $\mathrm{Q}$ are denoted by area $Q, \operatorname{per} Q$, and $\operatorname{col} Q$. In Diagram 3, area $Q=29$, per $Q=38$, and $\operatorname{col} Q=$ 8. The $k$ th column of $Q$ will be denoted by $Q_{k}$. We sometimes write $Q=Q_{1} Q_{2} \cdots Q_{\operatorname{col} Q}$.

The enumeration of CCPs and of subclasses of CCPs by various statistics has been widely studied. Polyomino enumeration is surveyed in Delest [10], Guttmann [19], Rensburg [35], and Viennot [39]. Our purpose here is to essentially initiate the study of CCPs by consecutive (or ridge) patterns.

\section{Diagram 3: A Column-Convex Polyomino}

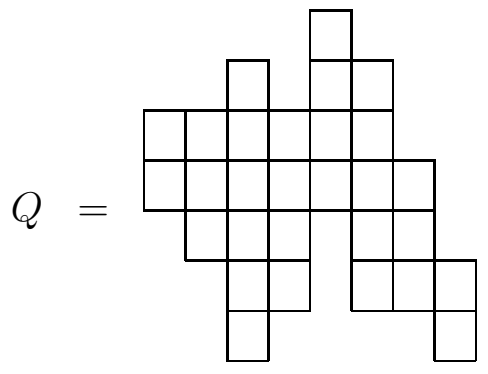

The simplest ridge patterns are formed between two adjacent columns. For a columnconvex polyomino $Q$, we say that an upper ascent (respectively upper level, upper descent) occurs at index $k$ if the top cell in $Q_{k}$ is lower than (respectively level with, higher than) the top cell in $Q_{k+1}$. Lower ascents, lower levels, and lower descents are similarly defined along the lower ridge. In Diagram 3, $Q$ has lower descents at indices 1,2,5, and 7 . The numbers of upper ascents, upper levels, upper descents, lower ascents, lower levels, and lower descents in $Q$ are respectively denoted by uasc $Q$, ulev $Q$, udes $Q$, lasc $Q, \operatorname{llev} Q$, and ldes $Q$. In Diagram 3, uasc $Q=2$ and $\operatorname{llev} Q=1$.

As displayed in Diagram 4, the two-column ridge patterns may be used to characterize many of the common subclasses of CCPs. 


\section{Diagram 4: Common Classes of CCPs.}

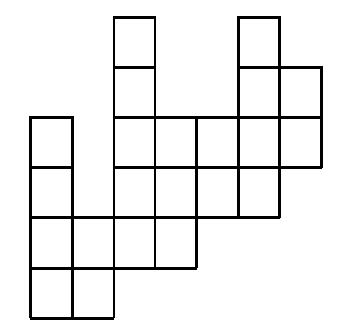

Directed Column-Convex Polyomino

(DCCP): No lower descents

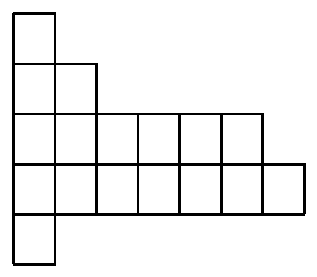

Stack Polyomino: No upper ascents and no lower descents

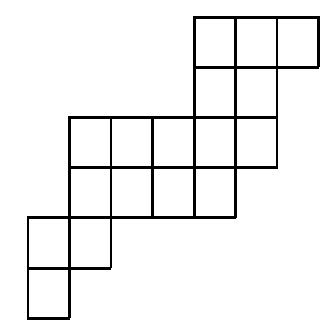

Parallelogram Polyomino:

No lower or upper descents

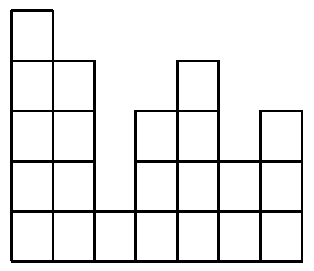

Wall Polyomino: No lower ascents and no lower descents

More complex consecutive patterns along either the lower or upper ridges are formed by segments of 3 or more columns.

The relative height of a $\operatorname{CCP} Q$, denoted by $\operatorname{relh} Q$, is defined to be the $y$-ordinate of the top edge in the rightmost column of $Q$. In Diagram $3, \operatorname{relh} Q=-1$. The relative height of a parallelogram polyomino is known as its row number.

\subsection{Verification that $\mathcal{P C} \subset \mathcal{P C C P} \subset \mathcal{P W}$}

Let $\mathrm{WP}_{n}$ be the set of wall polyominoes with $n$ columns. The map $\gamma_{n}: K_{n} \rightarrow \mathrm{WP}_{n}$ defined by $\gamma_{n}(w)=Q$ where $Q_{k}$ has $w_{k}$ cells is a bijection such that

$$
\text { area } Q=\operatorname{sum} w \text { and } \operatorname{per} Q-2 \operatorname{col} Q=\operatorname{var} w \text {. }
$$

For example, $\gamma_{7}$ maps the composition $w=5413423 \in K_{7}$ to the wall polyomino displayed in Diagram 4. Interestingly, the second part of (16) relates the variation of a composition to the perimeter of a wall polyomino, and (10) together with the first part of (16) provides a connection between the inversion number of a permutation and the area of a wall polyomino.

Through $\gamma_{n}$, a consecutive $p$-pattern in a composition $w$ induces an upper ridge $p$ pattern in the associated wall polyomino $Q$. For instance, $Q_{k} Q_{k+1} Q_{k+2}$ is deemed a 132-pattern in $Q$ if $w_{k} w_{k+1} w_{k+2}$ is a 132-pattern in the associated $w$; that is, $Q_{k} Q_{k+1} Q_{k+2}$ is a 132-pattern if $Q_{k+1} Q_{k+2}$ is an upper descent and if the top cell in $Q_{k+2}$ is level with or above the top cell in $Q_{k}$. The number of times an upper ridge pattern $p$ occurs in $Q$ is denoted by $p(Q)$. 
then

The bijection $\gamma_{n}$ immediately implies $\mathcal{P C} \subset \mathcal{P C C P}$ : If $P \subseteq \cup_{m \geqslant 1} S_{m}$ and if $B_{n} \subseteq K_{n}$,

$$
\sum_{n \geqslant 0} \sum_{w \in B_{n}} c^{\operatorname{var} w} q^{\text {sum } w}\left(\prod_{p \in P} y_{p}^{p(w)}\right) z^{n}=\sum_{n \geqslant 0} \sum_{Q \in \gamma_{n}\left(B_{n}\right)} c^{\text {per } Q} q^{\text {area } Q}\left(\prod_{p \in P} y_{p}^{p(Q)}\right) \frac{z^{n}}{c^{2 n}} .
$$

Of course, (17) also holds for maximal numbers of non-overlapping patterns.

To see that $\mathcal{P C C P} \subset \mathcal{P} \mathcal{W}$, consider the alphabet of biletters $X=\left\{\left(\begin{array}{c}j \\ m\end{array}\right): j, m \in N\right\}$ and let

$$
\mathcal{Y}=\bigcup_{n \geqslant 0}\left\{\left(\begin{array}{cccc}
j_{1} & j_{2} & \ldots & j_{n} \\
m_{1} & m_{2} & \ldots & m_{n}
\end{array}\right) \in X^{n}: m_{n}=1 \text { and } j_{k}+j_{k+1}>m_{k} \text { for } 1 \leqslant k<n\right\} .
$$

For a column-convex polyomino $\mathrm{Q}$ with $\mathrm{n}$ columns, define

$$
\delta(Q)=\left(\begin{array}{cccc}
j_{1} & j_{2} & \ldots & j_{n} \\
m_{1} & m_{2} & \ldots & m_{n}
\end{array}\right)
$$

where $j_{k}$ is the number of cells in $Q_{k}, m_{n}=1$, and, for $1 \leqslant k<n, m_{k}$ is the change in the $y$-ordinate from the bottom edge of $Q_{k+1}$ to the top edge of $Q_{k}$. For $Q$ in Diagram 3,

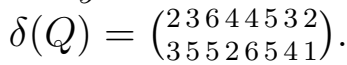

The map $\delta$ is a bijection from CCP to $\mathcal{Y}$. As such, $\delta$ allows CCPs to be viewed as words. Such a viewpoint is implicit in Temperley [37] and explicit in Bousquet-Mélou and Viennot [3]. Thus, a problem in $\mathcal{P C C P}$ may readily be converted into a problem in $\mathcal{P} \mathcal{W}$; so $\mathcal{P C C P} \subset \mathcal{P} \mathcal{W}$.

\section{Application of Theorem 3 to the set $\mathcal{P C C P}$}

The inclusion $\mathcal{P C C P} \subset \mathcal{P} \mathcal{W}$ means that Theorem 3 may be applied to solving problems in $\mathcal{P C C P}$. We present two examples on directed column-convex polyominoes.

\subsection{DCCPs by two-column ridge patterns}

Our first example enumerates DCCPs by the five two-column ridge patterns, perimeter, relative height, area, and column number.

Corollary 7. The generating function

$$
G=\sum_{Q \in \mathrm{DCCP}} a_{u}^{\text {uasc } Q} a_{l}^{\operatorname{lasc} Q} b_{u}^{\text {ulev } Q} b_{l}^{\text {llev } Q} c^{\operatorname{per} Q} d^{\operatorname{udes} Q} h^{\text {relh } Q} q^{\text {area } Q} z^{\operatorname{col} Q}
$$

is given by

$$
G=\frac{c^{2} h \sum_{n \geqslant 0} \frac{\left(c^{2} q z\right)^{n+1}}{1-c^{2} h q^{n+1}} \prod_{k=1}^{n}\left(b_{l}+\frac{a_{l} c^{2} h q^{k}}{1-c^{2} h q^{k}}\right)\left(b_{u}+\frac{c^{2} d q^{k}}{1-c^{2} q^{k}}-\frac{a_{u}}{1-q^{k}}\right)}{1-a_{u} \sum_{n \geqslant 1} \frac{\left(c^{2} q z\right)^{n}}{1-q^{n}} \prod_{k=1}^{n}\left(b_{l}+\frac{a_{l} c^{2} h q^{k}}{1-c^{2} h q^{k}}\right) \prod_{k=1}^{n-1}\left(b_{u}+\frac{c^{2} d q^{k}}{1-c^{2} q^{k}}-\frac{a_{u}}{1-q^{k}}\right)} .
$$


Proof. Define

$$
H\left(b_{u}, b_{l}, d, z\right)=\sum_{Q \in \mathrm{DCCP}} b_{u}^{\text {ulev } Q} b_{l}^{\text {llev } Q} c^{\text {per } Q} d^{\text {udes } Q} h^{\text {relh } Q} q^{\text {area } Q} z^{\operatorname{col} Q} .
$$

As uasc $Q=\operatorname{col} Q-\operatorname{ulev} Q-\operatorname{udes} Q-1$ and $\operatorname{lasc} Q=\operatorname{col} Q-\operatorname{llev} Q-1$, it follows that

$$
G=\frac{1}{a_{u} a_{l}} H\left(b_{u} / a_{u}, b_{l} / a_{l}, d / a_{u}, a_{u} a_{l} z\right) .
$$

It then suffices to determine $H$.

Consider $X=\left\{\left(\begin{array}{c}j \\ m\end{array}\right): j, m\right.$ integers, $\left.j \geqslant m \geqslant 1\right\}$. Let $R=\left\{\left(\begin{array}{c}j \\ m\end{array}\right) \in X: m=1\right\}$ and, for a statement $S$, let $\chi(S)$ be 1 if $S$ is true and 0 otherwise. An element $\left(\begin{array}{c}j_{1} j_{2} \ldots j_{n} \\ m_{1} m_{2} \ldots m_{n}\end{array}\right) \in X^{n}$ will be abbreviated by $\left(\begin{array}{c}j \\ m\end{array}\right)$; so the $k$ th letter in $\left(\begin{array}{c}j \\ m\end{array}\right)$ is $\left(\begin{array}{c}j \\ m\end{array}\right)_{k}=\left(\begin{array}{c}j_{k} \\ m_{k}\end{array}\right)$.

Let $\mathcal{F}=\left\{\left(\begin{array}{l}j \\ m\end{array}\right) \in X^{2}: m_{1} \geqslant j_{2}\right\}$. For $f=\left(\begin{array}{c}j \\ m\end{array}\right) \in \mathcal{F}$, set $y_{f}=c^{2\left(m_{1}-j_{2}\right)} d\left(b_{u} d^{-1}\right)^{\chi\left(m_{1}=j_{2}\right)}$. When restricted to DCCP, the map $\delta$ in (18) is a bijection onto $X^{*} R$. Moreover, if $Q=Q_{1} Q_{2} \ldots Q_{n} \in \operatorname{DCCP}$ and $\delta(Q)=\left(\begin{array}{c}j \\ m\end{array}\right) \in X^{n-1} R$, then

$$
\begin{gathered}
\operatorname{area} Q=\sum j, \text { per } Q=2(n+\operatorname{relh} Q+S), \\
\operatorname{relh} Q=\operatorname{sum} j-\operatorname{sum} m+1, \text { and } b_{u}^{\text {ulev } Q} d^{\text {udes } Q} c^{2 S}=\prod_{f \in \mathcal{F}} y_{f}^{f\left({ }_{m}^{j}\right)}
\end{gathered}
$$

where $S=\sum_{k=1}^{n}\left(m_{k}-j_{k+1}\right) \chi\left(m_{k}>j_{k+1}\right)$. The facts in (21) regarding area and relative height were observed by Bousquet-Mélou and Viennot [3].

It follows from (19) and (21) that

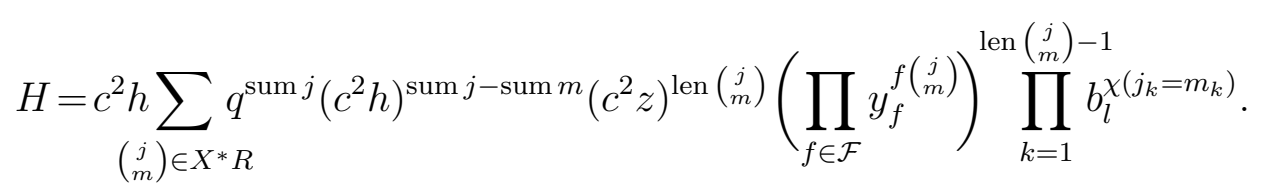

Note that an $\mathcal{F}$-cluster $\left(\left(\begin{array}{c}j \\ m\end{array}\right), \nu, \beta\right)$ has $\left(\begin{array}{c}j \\ m\end{array}\right) \in X^{n}, \nu=\left(\left(\begin{array}{c}j_{1} j_{2} \\ m_{1} m_{2}\end{array}\right),\left(\begin{array}{c}j_{2} j_{3} \\ m_{2} m_{3}\end{array}\right), \ldots,\left(\begin{array}{c}j_{n-1} j_{n} \\ m_{n-1} m_{n}\end{array}\right)\right)$, and $\beta=(1,2, \ldots, n-1)$ for some $n \geqslant 2$. So, application of Theorem 3 to (22) yields

$$
H=\frac{c^{2} h \sum_{n \geqslant 0}\left(c^{2} z\right)^{n+1} \sum_{T}(n)}{1-\sum_{n \geqslant 1}\left(c^{2} z\right)^{n} \sum_{B}(n)}
$$

where $\sum_{T}(n)$ is

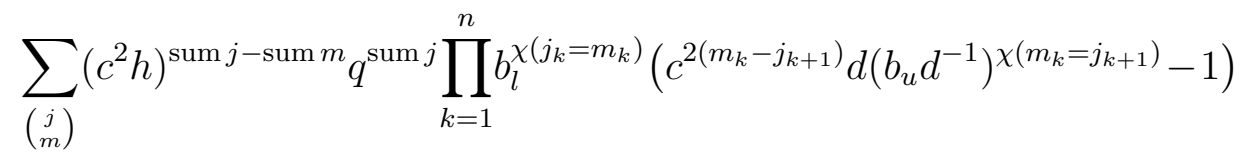

summed over $\left(\begin{array}{c}j \\ m\end{array}\right)$ satisfying $j_{1} \geqslant m_{1} \geqslant j_{2} \geqslant \ldots \geqslant j_{n+1} \geqslant m_{n+1}=1$ and $\sum_{B}(n)$ is

$$
\sum_{\left(\begin{array}{l}
j \\
m
\end{array}\right)}\left(c^{2} h\right)^{\operatorname{sum} j-\operatorname{sum} m} q^{\operatorname{sum} j} b_{l}^{\chi\left(j_{n}=m_{n}\right)} \prod_{k=1}^{n-1} b_{l}^{\chi\left(j_{k}=m_{k}\right)}\left(c^{2\left(m_{k}-j_{k+1}\right)} d\left(b_{u} d^{-1}\right)^{\chi\left(m_{k}=j_{k+1}\right)}-1\right)
$$


summed over $\left(\begin{array}{c}j \\ m\end{array}\right)$ satisfying $j_{1} \geqslant m_{1} \geqslant j_{2} \geqslant \ldots \geqslant j_{n} \geqslant m_{n} \geqslant 1 . \operatorname{Both} \sum_{T}(n)$ and $\sum_{B}(n)$ are nested geometric sums. As such, they are easily determined. For instance, $\sum_{T}(1)$ equals

$$
\begin{gathered}
q^{2} \sum_{j_{2} \geqslant 1}\left(c^{2} h q^{2}\right)^{j_{2}-1} \sum_{m_{1} \geqslant j_{2}} q^{m_{1}-j_{2}}\left(c^{2\left(m_{1}-j_{2}\right)} d\left(b_{u} d^{-1}\right)^{\chi\left(m_{1}=j_{2}\right)}-1\right) \sum_{j_{1} \geqslant m_{1}} b_{l}^{\chi\left(j_{1}=m_{1}\right)}\left(c^{2} h q\right)^{j_{1}-m_{1}} \\
=\frac{q^{2}}{1-c^{2} h q^{2}}\left(b_{u}+\frac{c^{2} d q}{1-c^{2} q}-\frac{1}{1-q}\right)\left(b_{l}+\frac{c^{2} h q}{1-c^{2} h q}\right) .
\end{gathered}
$$

In general,

$$
\begin{aligned}
& \sum_{T}(n)=\frac{q^{n+1}}{1-c^{2} h q^{n+1}} \prod_{k=1}^{n}\left(b_{l}+\frac{c^{2} h q^{k}}{1-c^{2} h q^{k}}\right)\left(b_{u}+\frac{c^{2} d q^{k}}{1-c^{2} q^{k}}-\frac{1}{1-q^{k}}\right) \text { and } \\
& \sum_{B}(n)=\frac{q^{n}}{1-q^{n}} \prod_{k=1}^{n}\left(b_{l}+\frac{c^{2} h q^{k}}{1-c^{2} h q^{k}}\right) \prod_{k=1}^{n-1}\left(b_{u}+\frac{c^{2} d q^{k}}{1-c^{2} q^{k}}-\frac{1}{1-q^{k}}\right) .
\end{aligned}
$$

The last two equalities for $\sum_{T}(n)$ and $\sum_{B}(n)$ together with (23) and (20) complete the proof.

Corollary 7 (with $a_{u}=a, b_{u}=b, a_{l}=0, b_{l}=h=1$, and $z$ replaced by $z / c^{2}$ ) with (17) implies Corollary 2 of subsection 3.3. Corollary 7 also implies many known results, a few of which are displayed in Table 1.

Table 1

\begin{tabular}{|c|c|c|}
\hline Polyominoes & Distribution & Reference \\
\hline \hline DCCP & $\begin{array}{c}\text { (area, per, relh, udes, col) } \\
a_{u}, a_{l}, b_{u}, b_{l}=1\end{array}$ & Rawlings [33] \\
\hline DCCP & $\begin{array}{c}(\text { area, per, relh, col }) \\
a_{u}, a_{l}, b_{u}, b_{l}, d=1\end{array}$ & Bousquet-Melou[4] \\
\hline $\mathrm{PP}$ & $\begin{array}{c}(\text { area, uasc, lasc, col) } \\
a_{u}, a_{l}, c, h=1 ; d=0\end{array}$ & $\begin{array}{c}\text { Delest, Dubernard, } \\
\text { and Dutour [12] }\end{array}$ \\
\hline $\mathrm{PP}$ & $\begin{array}{c}(\text { area, col }) \\
a_{u}, a_{l}, b_{u}, b_{l}, c, h=1 ; d=0\end{array}$ & Delest and Fédou [11] \\
\hline
\end{tabular}

The noted distribution of Delest, Dubernard, and Dutour [12] also tracked the area of the leftmost column; their notion of corners coincide exactly with upper and lower ascents. Bousquet-Mélou's entry included both the left and right column areas.

\subsection{DCCPs by valleys along the upper ridge}

A column-segment $Q_{k} Q_{k+1} Q_{k+2}$ in a column-convex polyomino $Q$ is said to be a valley provided that $Q_{k} Q_{k+1}$ is an upper descent and $Q_{k+1} Q_{k+2}$ is an upper ascent or an upper 
level. The number of valleys in $Q$ is denoted by $\operatorname{val}(Q)$. Furthermore, $Q$ is said to be downup provided that $Q_{k} Q_{k+1}$ is an upper descent when $k$ is odd and is an upper ascent or an upper level when $k$ is even. Let $\mathrm{DU}_{n}$ denote the set of down-up directed column-convex polyominoes of length $n$.

Corollary 8. The generating function for DCCPs by valleys, area, and column number is

$$
\sum_{Q \in \mathrm{DCCP}} y^{\operatorname{val}(Q)} q^{\operatorname{area} Q} z^{\operatorname{col} Q}=\frac{\sum_{n \geqslant 0} \frac{(1-y)^{n} q^{(n+1)(2 n+1)} z^{2 n+1}}{(q ; q)_{2 n+1}(q ; q)_{2 n}}}{\sum_{n \geqslant 0} \frac{(1-y)^{n} q^{n(2 n+1)} z^{2 n}}{(q ; q)_{2 n}^{2}}-\sum_{n \geqslant 0} \frac{(1-y)^{n} q^{(n+1)(2 n+1)} z^{2 n+1}}{(q ; q)_{2 n+1}^{2}}} .
$$

The proof of Corollary 8 consists of first using Theorem 3 to express the generating function for DCCPs by valleys in terms of down-up DCCPs of odd lengths. Theorem 3 is then applied again in a manner analogous to the second half of the proof of Corollary 3 to show that

$$
\sum_{n \geqslant 0} \sum_{Q \in \mathrm{DU}_{2 n+1}} q^{\text {area } Q} z^{2 n+1}=\left(\sum_{n \geqslant 0} \frac{(-1)^{n} q^{(n+1)(2 n+1)} z^{2 n+1}}{(q ; q)_{2 n+1}(q ; q)_{2 n}}\right)\left(\sum_{n \geqslant 0} \frac{(-1)^{n} q^{n(2 n+1)} z^{2 n}}{(q ; q)_{2 n}^{2}}\right)^{-1} .
$$

\section{The Pattern Algebra and Q1}

Goulden and Jackson's Pattern Algebra [18, section 4.3] is a powerful method for solving the composition version of Q1 for a pattern set, tracked as a whole, of the form $P=\{p \in$ $\left.S_{m}: p_{1} *_{1} p_{2} *_{2} \ldots *_{m-1} p_{m}\right\}$ where $*_{1}, *_{2}, \cdots, *_{m-1}$ belong to a bipartition of $N^{2}$. In this section, we use their Pattern Algebra to obtain a $q$-analog of Kitaev's [25] Theorem 30 and to deduce a better generating function for permutations by peaks and twin peaks.

The essentials of the Pattern Algebra follow. Let $X$ be an alphabet, $\pi_{1} \subset X^{2}$, and $\pi_{2}=X^{2} \backslash \pi_{1}$. Suppose $\alpha=\sum_{w \in X^{*}} c_{w} w$ is a formal series where the constants commute with letters of $X$, and for given $x, y \in X$, let $X_{x, y}=\left\{w \in X^{+}: w_{1}=x, w_{\text {len } w}=y\right\}$. Then, the incidence matrix $I(\alpha)$ is a matrix with rows and columns indexed by $X$ such that $I(\alpha)_{x, y}=\sum_{w \in X_{x, y}} c_{w}(w / y)$, i.e. the restriction of $\alpha$ to words in $X_{x, y}$, except the final $y$ has been removed from each word. For $U \subseteq X^{*}$, we also define $I(U)=I\left(\sum_{w \in U} w\right)$ and note that $I(X)=I$, the identity matrix.

For the remainder of this section, we let $A=I\left(\pi_{1}\right), B=I\left(\pi_{2}\right)$, and $W=I\left(X^{2}\right)$. In particular, $W=A+B$. It is crucial to note that, for formal series $\alpha$ and $\beta, I(\alpha) I(\beta)=$ $I(\gamma)$, where $\gamma$ is formed by concatenating words $u$ and $v$ from $\alpha$ and $\beta$, respectively, where the last letter of $u$ is the first letter of $v$, and removing one copy of the repeated letter.

Finally, we define the operator $\Psi$, which converts an incidence matrix back to a formal series, by $\Psi\left(I\left(\sum_{w \in X^{*}} c_{w} w\right)\right)=\sum_{w \in X^{+}} c_{w} w$. The empty word has been removed in the process, as it is not accounted for in the incidence matrix. Note that $\Psi$ is linear and, for incidence matrices $F$ and $G, \Psi(F W G)=\Psi(F) \Psi(G)$. 


\subsection{A General Strategy}

We will consider the problem of enumerating words by pattern sets whose incidence matrices can be written as a rational function of $A, W$, and $B$. Such a problem can be solved by the following process, which is distinct, yet equal in scope, to that given by Goulden and Jackson.

1. Define a variable to be the incidence matrix for the desired formal series, and then devise a system of linear equations to describe it. The design of this system should mimic that of a regular grammar in that each variable will be multiplied by at most one of $A, W$, and $B$ and always on the same side. We will name our variables $F_{i}$ and use right multiplication in this section.

2. Substitute either $A=W-B$ or $B=W-A$ and solve the system, treating $F_{i} W$ terms as constants. Using $B=W-A$, we obtain a system of the form $F_{i}=f_{i}(A)+\sum_{j} F_{j} W f_{i j}(A)$, where the $f_{\mathrm{s}}$ are rational functions.

3. Finally, apply $\Psi$ to the entire system, noting that $\Psi(F W G)=\Psi(F) \Psi(G)$, and solve for $\Psi\left(F_{i}\right)$.

We will typically compute $\Psi(f(A))$ by expanding $f(A)$ as a power series in $A$ and using the linearity of $\Psi$ to get a formal sum of the $\Psi\left(A^{n}\right)$. We then may apply homomorphisms to the solution to obtain various generating functions. We compute the image of $\Psi\left(A^{n}\right)$ under some common homomorphisms in the next subsection.

For DCCPs, we must also compute $\Psi\left(F_{i} Z\right)$, where $Z$ is an incidence matrix that restricts the last letter of each word. We achieve this by multiplying the equation for $F_{i}$ by $Z$ before the final step and using known values of $\Psi\left(F_{i}\right)$. We will then also need to compute the image of $\Psi\left(A^{n} Z\right)$.

\subsection{Key formulas}

As $\Psi\left(A^{n}\right)$ and $\Psi\left(B^{n}\right)$ show up frequently, it is prudent to give their values under a few common homomorphisms.

Recall that $N=\{1,2,3, \ldots\}$. For $\pi_{1}=\{i j: i \leqslant j\}$ and $\phi_{N}$ defined on $N^{*}$ by $\phi_{N}(w)=q^{\operatorname{sum} w}(z / q)^{\text {len } w}$, well-known partition identities imply that

$$
\phi_{N}\left(\Psi\left(A^{n-1}\right)\right)=z^{n} /(q ; q)_{n} \text { and } \phi_{N}\left(\Psi\left(B^{n-1}\right)\right)=z^{n} q^{\left(\begin{array}{c}
n \\
2
\end{array}\right)} /(q ; q)_{n} .
$$

Next, consider the alphabets $X=\left\{\left(\begin{array}{c}j \\ m\end{array}\right): j, m\right.$ integers, $\left.j \geqslant m \geqslant 1\right\}$ and $R=\left\{\left(\begin{array}{c}j \\ m\end{array}\right) \in\right.$ $X: m=1\}$. Let $\pi_{1}=\left\{\left(\begin{array}{c}j_{1} j_{2} \\ m_{1} m_{2}\end{array}\right): j_{2} \geqslant m_{1}\right\}$. Define $\phi_{X}$ to be the homomorphism that maps $\left(\begin{array}{c}j \\ m\end{array}\right)$, where $j, m \in N^{*}$, to $q^{\text {sum } j} z^{\text {len } j}$. Then $\phi_{X}\left(\Psi\left(B^{n-1}\right)\right)$ is the sum of $q^{\text {sum } j} z^{n}$ over $\left(\begin{array}{c}j \\ m\end{array}\right) \in X^{n}$ where $0<m_{n} \leqslant j_{n}<m_{n-1} \leqslant j_{n-1}<\cdots<m_{1} \leqslant j_{1} . \phi_{X}\left(\Psi\left(B^{n-1} I(R)\right)\right)$ is the same sum as $\phi_{X}\left(\Psi\left(B^{n-1}\right)\right)$, except $m_{n}=1$. Thus,

$$
\phi_{X}\left(\Psi\left(B^{n-1}\right)\right)=\frac{q^{\left(\begin{array}{c}
n+1 \\
2
\end{array}\right)}}{(q ; q)_{n}^{2}} \text { and } \phi_{X}\left(\Psi\left(B^{n-1} I(R)\right)\right)=\frac{q^{\left(\begin{array}{c}
n+1 \\
2
\end{array}\right)}}{(q ; q)_{n}(q ; q)_{n-1}} \text {. }
$$


The sums $\phi_{X}\left(\Psi\left(A^{n-1}\right)\right)$ and $\phi_{X}\left(\Psi\left(A^{n-1} I(R)\right)\right)$ are more difficult to compute. Theorem 5 addresses this issue.

Theorem 5. Given $A(x)=1+\Psi\left(A^{0}\right) x+\Psi\left(A^{1}\right) x^{2}+\cdots, B(x)=1+\Psi\left(B^{0}\right) x+\Psi\left(B^{1}\right) x^{2}+$ $\cdots, A Z(x)=\Psi(Z)+\Psi(A Z) x+\Psi\left(A^{2} Z\right) x^{2}+\cdots$, and $B Z(x)=\Psi(Z)+\Psi(B Z) x+$ $\Psi\left(B^{2} Z\right) x^{2}+\cdots$, then $A(x)=(B(-x))^{-1}$ and $A Z(x)=A(x) B Z(-x)$.

Proof. Let $F=x(I-x A)^{-1}$, so that $A(x)=1+\Psi(F)$. Then, $F-x F A=x I$. Set $A=W-B$ and solve for $F$, treating $F W$ as a constant. It follows that

$$
F=x(I+x B)^{-1}+x F W(I+x B)^{-1} .
$$

Applying $\Psi$ and using the fact that $\Psi(F W G)=\Psi(F) \Psi(G)$ yields

$$
\Psi(F)=x \Psi\left((I+x B)^{-1}\right)+x \Psi(F) \Psi\left((I+x B)^{-1}\right) .
$$

Factor, add 1 to both sides, and solve for $1+\Psi(F)$ to obtain

$$
1+\Psi(F)=\left(1-x \Psi\left((I+x B)^{-1}\right)\right)^{-1}=(B(-x))^{-1} .
$$

Now, let $G=(I-x A)^{-1} Z$, so that $A Z(x)=\Psi(G)$. Then, $G-x A G=Z$. Set $A=W-B$ and solve for $G$, treating $W G$ as a constant. It follows that

$$
G=(I+x B)^{-1} Z+x(I+x B)^{-1} W G .
$$

Applying $\Psi$ and using the fact that $\Psi(F W G)=\Psi(F) \Psi(G)$ yields

$$
\Psi(G)=\Psi\left((I+x B)^{-1} Z\right)+x \Psi\left((I+x B)^{-1}\right) \Psi(G) .
$$

Finally, solve for $\Psi(G)$ and note that $\left(1-x \Psi\left((I+x B)^{-1}\right)\right)^{-1}=(B(-x))^{-1}=A(x)$ and $\Psi\left((I+x B)^{-1} Z\right)=B Z(-x)$ to complete the result.

Applying Theorem 5, we find that

$$
\begin{gathered}
\phi_{X}\left(\Psi\left(A^{n-1}\right)\right)=\left.\left(\sum_{k \geqslant 0} \frac{(-x)^{k} q^{\left(\begin{array}{c}
k+1 \\
2
\end{array}\right)}}{(q ; q)_{k}^{2}}\right)^{-1}\right|_{x^{n}} \text { and that } \\
\phi_{X}\left(\Psi\left(A^{n-1} I(R)\right)\right)=\left.\left(\sum_{k \geqslant 0} \frac{(-x)^{k} q^{\left(\begin{array}{c}
k+2 \\
2
\end{array}\right)}}{(q ; q)_{k+1}(q ; q)_{k}}\right)\left(\sum_{k \geqslant 0} \frac{(-x)^{k} q^{\left(\begin{array}{c}
k+1 \\
2
\end{array}\right)}}{(q ; q)_{k}^{2}}\right)^{-1}\right|_{x^{n-1}} .
\end{gathered}
$$

Note that (26) counts the number of parallelogram polyominoes with $n$ columns by area, matching the result of Delest and Fédou [11]. 


\subsection{A $q$-analog of a distribution due to Kitaev}

In this section, we use the Pattern Algebra to obtain a $q$-analog of Kitaev's Theorem 30 in [25] that enumerates permutations that avoid $P=\{4312,4213,4123,3214,3124,2134\}$. We begin by deducing the relevant generating function on words.

Theorem 6. If $\mathcal{F} \subset X^{4}$ such that $I(\mathcal{F})=A W B$, then

$$
\sum_{w \in X^{*}} y^{\mathcal{F}(w)} w=\left(1-\alpha \beta^{-1}\right)^{-1}
$$

where $\mathcal{F}(w)=\sum_{f \in \mathcal{F}} f(w)$ and

$$
\begin{aligned}
\alpha & =\Psi(U)-\Psi(B U) \gamma \\
\beta & =1+(y-1)^{2} \Psi\left(B^{3} U\right)-\Psi(U) \gamma \\
\gamma & =(y-1)\left(1+(y-1) \Psi\left(B^{2} U+(y-1) B^{3} U\right)\right)^{-1} \Psi\left(B U+(y-1) B^{2} U\right) \\
U & =\left(I-(y-1)^{2} B^{4}\right)^{-1} .
\end{aligned}
$$

Proof. For all $f \in \mathcal{F}$, let $y_{f}=y$, and let $F_{0}=I(X)+I\left(C_{\mathcal{F}}\left(\mathbf{y}-\mathbf{1}, X^{*}\right)\right)$, where $C_{\mathcal{F}}$ is the cluster generating function as defined in section 4. By Theorem 3, we then seek to compute $\left(1-\Psi\left(F_{0}\right)\right)^{-1}$. Following our general strategy in subsection 8.1 , we construct the system:

$$
\begin{array}{ll}
F_{0}=I+(y-1) F_{2} B+(y-1) F_{3} B & F_{2}=F_{1} A \\
F_{1}=F_{0} A & F_{3}=F_{1} B+(y-1) F_{2} B .
\end{array}
$$

Next, we substitute $A=W-B$ and, since there were no $F_{2} A$ or $F_{3} A$ terms in the system, immediately eliminate $F_{2}$ and $F_{3}$, giving

$$
F_{0}=I+(y-1) F_{1} W\left(B+(y-1) B^{2}\right)-(y-1)^{2} F_{1} B^{3} \text { and } F_{1}=F_{0} W-F_{0} B .
$$

Treating $F_{0} W$ and $F_{1} W$ as constants, solve for $F_{0}$ and $F_{1}$ to get

$$
\begin{aligned}
& F_{0}=\left(I+(y-1) F_{1} W\left(B+(y-1) B^{2}\right)-(y-1)^{2} F_{0} W B^{3}\right) U \text { and } \\
& F_{1}=\left(F_{0} W-B-(y-1) F_{1} W\left(B^{2}+(y-1) B^{3}\right)\right) U
\end{aligned}
$$

Using the property $\Psi(F W G)=\Psi(F) \Psi(G)$, take $\Psi$ of both sides to obtain

$$
\begin{aligned}
& \Psi\left(F_{0}\right)=\Psi(U)+(y-1) \Psi\left(F_{1}\right) \Psi\left(B U+(y-1) B^{2} U\right)-(y-1)^{2} \Psi\left(F_{0}\right) \Psi\left(B^{3} U\right) \\
& \Psi\left(F_{1}\right)=\Psi\left(F_{0}\right) \Psi(U)-\Psi(B U)-(y-1) \Psi\left(F_{1}\right) \Psi\left(B^{2} U+(y-1) B^{3} U\right) .
\end{aligned}
$$

Finally, solve the system for $\Psi\left(F_{0}\right)$ and compute $\left(1-\Psi\left(F_{0}\right)\right)^{-1}$.

Recall $\sin _{q} z$ and $\cos _{q} z$ of subsection 2.1. Let $e_{q}(z)=\sum_{n \geqslant 0} z^{n} /(q ; q)_{n}$. The generating function $\mathcal{K}$ in the following result gives a $q$-analog of Theorem 30 in [25]. 
Corollary 9. If $P=\{1243,1342,1432,2341,2431,3421\}$, then

$$
\sum_{n \geqslant 0} \sum_{\sigma \in S_{n}} \frac{y^{P_{n o}(\sigma)} q^{\text {inv } \sigma} z^{n}}{(q ; q)_{n}}=\frac{\mathcal{K}}{1-y+y\left(1-\frac{z}{1-q}\right) \mathcal{K}}
$$

where

$$
\mathcal{K}=\frac{e_{q}(z) e_{q}(-z)+\cos _{q}^{2} z+\sin _{q}^{2} z+2 e_{q}(-z) \cos _{q} z+\left(e_{q}(z)+e_{q}(-z)\right) \sin _{q} z}{4 e_{q}(-z) \cos _{q} z} .
$$

Proof. Let $T=\{4312,4213,4123,3214,3124,2134\}$. Then, the "complement-reversal" bijection $c_{n}: S_{n} \rightarrow S_{n}$ given by $c_{n}\left(\sigma_{1} \sigma_{2} \ldots \sigma_{n}\right)=\left(n+1-\sigma_{n}\right)\left(n+1-\sigma_{n-1}\right) \ldots\left(n+1-\sigma_{1}\right)$ shows that $\sum_{\sigma \in S_{n}} y^{P(\sigma)} q^{\text {inv } \sigma}=\sum_{\sigma \in S_{n}} y^{T(\sigma)} q^{\text {inv } \sigma}$. By Theorem 2,

$$
\sum_{n \geqslant 0} \sum_{\sigma \in S_{n}} \frac{y^{T(\sigma)} q^{\text {inv } \sigma} z^{n}}{(q ; q)_{n}}=\sum_{n \geqslant 0} \sum_{w \in K_{n}} y^{T(w)} q^{\text {sum } w}(z / q)^{n} .
$$

Using $\pi_{1}=\{i j: i>j\}$, note that $B^{n}$ is equivalent to what $A^{n}$ is when $\pi_{1}=\{i j: i \leqslant$ $j$ \}. The right-hand side of (27) may then be evaluated by applying (24) to the result in Theorem 6. Finally, set $y=0$ and apply Corollary 1 .

\subsection{The twin peak problem revisited}

Define a peak to be a single word from $\Psi(A B)$ and a twin peak to be one from $\Psi(A B A B)$. For $w \in X^{*}$, let $\operatorname{pic}(w)$ be the number of peaks in $w$ and $\operatorname{tpic}(w)$ be the number of twin peaks. Theorem 7 solves Q2 on words for peaks and twin peaks; as corollaries, we obtain corresponding results on permutations and DCCPs.

Theorem 7. For any nonempty alphabet $X$,

$$
\sum_{w \in X^{*}} x^{\operatorname{pic}(w)} y^{\operatorname{tpic}(w)} w=\left(1-\alpha \beta^{-1}\right)^{-1}
$$

where

$$
\begin{aligned}
\alpha & =\Psi(U)+x \Psi\left(B^{2} U\right)(y-1-\gamma), \\
\beta & =1+x(y-1) \Psi\left(B^{3} U\right)-\Psi(B U)(x-1+x \gamma), \\
\gamma & =x(y-1)\left(1-x(y-1) \Psi\left(B U-B^{3} U\right)\right)^{-1} \Psi(B U), \\
U & =\left(I+(x y-1) B^{2}-x(y-1) B^{4}\right)^{-1} .
\end{aligned}
$$

Proof. Let $\mathcal{F}$ be the set of peaks and twin peaks, $y_{f}=x$ if $f \in \mathcal{F}$ is a peak and $y_{f}=y$ if $f$ is a twin peak. Then, let $F_{0}=I(X)+I\left(C_{\mathcal{F}}\left(\mathbf{y}-\mathbf{1}, X^{*}\right)\right)$, where $C_{\mathcal{F}}$ is the cluster generating function of section 4 . By Theorem 3 , we then seek to compute $\left(1-\Psi\left(F_{0}\right)\right)^{-1}$. Following our general strategy in subsection 8.1 , we deduce a system of equations:

$$
\begin{array}{ll}
F_{0}=I+(x-1) F_{1} B+x(y-1) F_{3} B & F_{2}=x F_{1} B+x(y-1) F_{3} B \\
F_{1}=F_{0} A & F_{3}=F_{2} A .
\end{array}
$$


Substitute $A=W-B$ and eliminate $F_{1}$ and $F_{3}$ to obtain

$$
\begin{aligned}
& F_{0}=I+(x-1) F_{0} W B-(x-1) F_{0} B^{2}+x(y-1) F_{2} W B-x(y-1) F_{2} B^{2} \\
& F_{2}=x F_{0} W B-x F_{0} B^{2}+x(y-1) F_{2} W B-x(y-1) F_{2} B^{2} .
\end{aligned}
$$

Treating $F_{0} W$ and $F_{2} W$ as constants, solve for $F_{0}$ and $F_{2}$ :

$$
\begin{aligned}
& F_{0}=\left(I+x(y-1) B^{2}+F_{0} W\left((x-1) B-x(y-1) B^{3}\right)+x(y-1) F_{2} W B\right) U \\
& F_{2}=x\left(-B^{2}+F_{0} W B+(y-1) F_{2} W\left(B-B^{3}\right)\right) U .
\end{aligned}
$$

Apply $\Psi$, recall that $\Psi(F W G)=\Psi(F) \Psi(G)$, and solve for $\Psi\left(F_{0}\right)$.

When deriving a result for permutations, we generally prefer to set $B=W-A$ instead of $A=W-B$, since $\phi_{N}\left(\Psi\left(A^{n}\right)\right)$ is nicer than $\phi_{N}\left(\Psi\left(B^{n}\right)\right)$. However, as the following paragraph shows, when a homomorphism $\phi$ whose image is commutative is applied to Theorem 7, $A$ and $B$ may be switched without affecting the result.

Let $\mathcal{F}^{\prime}$ be the set of words from $\Psi(B A+B A B A), y_{f}=x$ if $f$ is from $\Psi(B A)$ and $y_{f}=y$ if $f$ is from $\Psi(B A B A)$. Let $(w, \nu, \beta)$ be an $\mathcal{F}$-cluster with $w \in X^{n}$. Then, if $w_{n} w_{1} \in \pi_{1}$, let $w^{\prime}=w_{2} w_{3} \ldots w_{n} w_{1}$; otherwise, let $w^{\prime}=w_{n} w_{1} w_{2} \ldots w_{n-1} . \quad\left(w^{\prime}, \nu^{\prime}, \beta\right)$ is then an $\mathcal{F}^{\prime}$-cluster, where $\nu^{\prime}$ contains factors of $w^{\prime}$ with lengths and positions matching those in $\nu$. Moreover, since no other word of the form $w_{i} \ldots w_{n} w_{1} \ldots w_{i-1}$ is $\mathcal{F}^{\prime}$-coverable, this is a bijection. Then, $\phi\left(C_{\mathcal{F}}\left(\mathbf{y}, \mathbf{X}^{*}\right)\right)=\phi\left(\mathbf{C}_{\mathcal{F}^{\prime}}\left(\mathbf{y}, \mathbf{X}^{*}\right)\right)$, since $w^{\prime}$ is a rearrangement of the letters of $w$.

Using the above fact, we obtain the following alternative solution to the problem considered in subsection 5.3.

Corollary 10. The generating function for permutations by peaks, twin peaks, and inversions is

$$
\sum_{n \geqslant 0} \sum_{\sigma \in S_{n}} \frac{q^{\operatorname{inv} \sigma} x^{\mathrm{pic}(\sigma)} y^{\mathrm{tpic}(\sigma)} z^{n}}{(q ; q)_{n}}=\left(1-\frac{s_{+} \sin _{q}\left(z \sqrt{r_{+}}\right)}{2 \sqrt{r_{+}} \cos _{q}\left(z \sqrt{r_{+}}\right)}-\frac{s_{-} \sin _{q}\left(z \sqrt{r_{-}}\right)}{2 \sqrt{r_{-}} \cos _{q}\left(z \sqrt{r_{-}}\right)}\right)^{-1}
$$

where $r_{ \pm}=(x y-1 \pm \sqrt{D}) / 2, s_{ \pm}=1 \pm(2 x-x y-1) / \sqrt{D}$, and $D=(x y+1)^{2}-4 x$.

Proof. Apply $\phi_{N}$ to Theorem 7, replace each $B$ with an $A$, expand $U$ as a series, then apply (24) and simplify.

A peak in a CCP $Q$ is a sequence of columns $Q_{i} Q_{i+1} Q_{i+2}$ such that $Q_{i} Q_{i+1}$ is an upper ascent or level and $Q_{i+1} Q_{i+2}$ is an upper descent. A twin peak is a sequence $Q_{i} Q_{i+1} Q_{i+2} Q_{i+3} Q_{i+4}$ such that $Q_{i} Q_{i+1} Q_{i+2}$ and $Q_{i+2} Q_{i+3} Q_{i+4}$ are peaks. Let pic $(Q)$ and $\operatorname{tpic}(Q)$ respectively denote the numbers of peaks and twin peaks in $Q$. Corollary 11 applies Theorem 7 to DCCPs.

Corollary 11. The generating function for DCCPs by peaks, twin peaks, area, and column number is

$$
\sum_{Q \in \operatorname{DCCP}} x^{\operatorname{pic}(Q)} y^{\operatorname{tpic}(Q)} q^{\operatorname{area} Q} z^{\operatorname{col} Q}=\frac{\phi_{X}\left(\Psi\left(F_{0} Z\right)\right)}{1-\phi_{X}\left(\Psi\left(F_{0}\right)\right)}
$$


where $Z=I(R), F_{0}$ and $U$ are as in Theorem 7 , and

$$
\begin{aligned}
\Psi\left(F_{0} Z\right)= & \Psi\left(U Z+x(y-1) B^{2} U Z\right)+\Psi\left(F_{0}\right) \Psi\left((x-1) B U Z-x(y-1) B^{3} U Z\right) \\
& +x(y-1) \Psi\left(F_{2}\right) \Psi(B U Z), \\
\Psi\left(F_{2}\right)= & x\left(-\Psi\left(B^{2} U\right)+\Psi\left(F_{0}\right) \Psi(B U)\right)\left(1-x(y-1) \Psi\left(B U-B^{3} U\right)\right)^{-1} .
\end{aligned}
$$

Proof. The bijection $\delta$ of (18), $\phi_{X}$ and $R$ of subsection 8.2, and Theorem 3 imply that we seek $\phi_{X}\left(\left(1-\Psi\left(F_{0}\right)\right)^{-1} \Psi\left(F_{0} Z\right)\right)$. To evaluate $\Psi\left(F_{0} Z\right)$, right-multiply both sides of the first equality in $(28)$ by $Z$, apply $\Psi$, and solve.

\section{$9 \quad$ The Temperley method}

In short, the Temperley [37] method involves introducing a variable to track the size of the last letter in a word, deriving a recurrence for the generating function using that variable, and then solving the recurrence. By streamlining Temperley's method, Bousquet-Mélou [4] developed a powerful tool for enumerating CCPs by practically any desired set of statistics. In this final section, we illustrate the use of the Temperley method as modified in [4] in solving a few select consecutive pattern problems on permutations, compositions, and CCPs. Theorems 3 and 4 also play a role.

\subsection{Compositions by mesas}

A mesa in a composition $w$ is a segment $w_{i} w_{i+1} w_{i+2} w_{i+3}$ satisfying $w_{i}<w_{i+1}=w_{i+2}>$ $w_{i+3}$. Let $\operatorname{mes}(w)$ be the number of mesas in $w$.

Corollary 12. The generating function for compositions by mesas is

$$
\sum_{n \geqslant 0} \sum_{w \in K_{n}} y^{\operatorname{mes}(w)} q^{\operatorname{sum} w}(z / q)^{n}=\left(1-\frac{\sum_{n \geqslant 0} \frac{(-1)^{n}(y-1)^{n} z^{3 n+1} q^{3 n(n+1) / 2}}{\left(q ; q^{3}\right)_{n+1}\left(q^{3} ; q^{3}\right)_{n}}}{\sum_{n \geqslant 0} \frac{(-1)^{n}(y-1)^{n} z^{3 n} q^{n(3 n+1) / 2}}{\left(q^{2} ; q^{3}\right)_{n}\left(q^{3} ; q^{3}\right)_{n}}}\right)^{-1} .
$$

Proof. Let $\mathcal{F}$ be the set of mesas. Set $y_{f}=y$ for $f \in \mathcal{F}$. Let $\phi(w)=q^{\operatorname{sum} w}(z / q)^{\operatorname{len} w} b^{w_{\text {len } w}-1}$ and let $F_{0}(b)=\frac{z}{1-q b}+\phi\left(C_{\mathcal{F}}\left(\mathbf{y}-\mathbf{1}, N^{*}\right)\right)$ where $C_{\mathcal{F}}\left(\mathbf{y}, N^{*}\right)$ is as in section 4 . By Theorem 3 , we seek $\left(1-F_{0}(1)\right)^{-1}$.

As with the Pattern Algebra, we deduce a system of equations. Note that $F_{0}$ is the sum of the weights of the one-letter and $\mathcal{F}$-coverable compositions. Let $F_{1}$ be the sum over those with an extra ascent at the end and $F_{2}$ be the sum over those with an extra ascent and level at the end. Then,

$$
\begin{aligned}
& F_{0}(b)=\frac{z}{1-q b}+\frac{(y-1) z}{1-q b}\left(F_{2}(1)-F_{2}(q b)\right), \\
& F_{1}(b)=\frac{z}{1-q b} F_{0}(q b), \text { and } F_{2}(b)=z F_{1}(q b) .
\end{aligned}
$$


Elimination of $F_{1}(b)$ and $F_{2}(b)$ gives

$$
F_{0}(b)=\frac{z}{1-q b}+\frac{(y-1) z^{3} q^{2}}{\left(1-q^{2}\right)(1-q b)} F_{0}\left(q^{2}\right)-\frac{(y-1) z^{3} q^{3} b}{(1-q b)\left(1-q^{3} b\right)} F_{0}\left(q^{3} b\right) .
$$

Iteration of this recurrence leads to

$$
F_{0}(b)=\left(z+\frac{(y-1) z^{3} q^{2}}{1-q^{2}} F_{0}\left(q^{2}\right)\right) \sum_{n \geqslant 0} \frac{(-1)^{n}(y-1)^{n} z^{3 n} q^{3 n(n+1) / 2} b^{n}}{\left(q b ; q^{3}\right)_{n+1}\left(q^{3} b ; q^{3}\right)_{n}} .
$$

Substituting $b=q^{2}$ and then solving yields

$$
z+\frac{(y-1) z^{3} q^{2}}{1-q^{2}} F_{0}\left(q^{2}\right)=\frac{z}{\sum_{n \geqslant 0} \frac{(-1)^{n}(y-1)^{n} z^{3 n} q^{n(3 n+1) / 2}}{\left(q^{2} ; q^{3}\right)_{n}\left(q^{3} ; q^{3}\right)_{n}}} .
$$

Substituting the above into (29) and setting $b=1$ completes the proof.

\subsection{Permutations by $(\mathrm{i}, \mathrm{m})$-maxima revisited}

The modified Temperley method in [4] may be applied to obtain an alternative version of the generating function in Corollary 5 .

Corollary 13. If $i \geqslant 2$ and $1 \leqslant m \leqslant i$, then the generating function for permutations by $(i, m)$-maxima and inversions is

$$
\sum_{n \geqslant 0} \sum_{\sigma \in S_{n}}\left(\prod_{m=1}^{i} y_{m}^{p_{(m)}(\sigma)}\right) \frac{q^{\mathrm{inv} \sigma} z^{n}}{(q ; q)_{n}}=\left(1-\frac{\sum_{n \geqslant 0} \frac{z^{i n+1}}{1-q^{i n+1}} \prod_{k=0}^{n-1} T\left(q^{i k}\right)}{1-\frac{y_{i}-1}{(q ; q)_{i-1}} \sum_{n \geqslant 1} \frac{z^{i n}}{1-q^{i n}} \prod_{k=1}^{n-1} T\left(q^{i k-1}\right)}\right)^{-1}
$$

where

$$
T(b)=\sum_{m=1}^{i-1} \frac{\left(y_{m}-1\right) q^{m}}{(q ; q)_{m}\left(q^{m+1} b ; q\right)_{i-m}}-\frac{y_{i}-1}{(q ; q)_{i-1}(1-q b)} .
$$

Proof. Define $F(b)=\frac{z}{1-q b}+\phi\left(C_{\mathcal{F}}\left(\mathbf{y}-\mathbf{1}, N^{*}\right)\right)$, where $\mathcal{F}=\cup_{p \in P_{i}} \mathcal{F}_{p}$ and $\phi$ is as in Corollary 12. By Theorem 4 , we seek $(1-F(1))^{-1}$. As usual, we derive a recurrence for $F(b)$. Note that $(i, m)$-maxima can never overlap non-trivially, so the only way to extend an $\mathcal{F}$-cluster is to append an $(i, m)$-maximum. For $1 \leqslant m<i$, the generating function for words from $F(b)$ with an $(i, m)$-maximum appended is

$$
\frac{\left(y_{m}-1\right) q^{m} z^{i}}{(q ; q)_{m}\left(q^{m+1} b ; q\right)_{i-m}} F\left(q^{i} b\right) .
$$


The generating function for words from $F(b)$ with an extra $(i, i)$-maximum appended is

$$
\frac{\left(y_{i}-1\right) z^{i}}{(q ; q)_{i-1}(1-q b)}\left(F\left(q^{i-1}\right)-F\left(q^{i} b\right)\right) .
$$

Summing (30) over $m$ and adding in (31) and the one letter words, we obtain a recurrence for $F(b)$ :

$$
F(b)=\frac{z}{1-q b}+\frac{\left(y_{i}-1\right) z^{i}}{(q ; q)_{i-1}(1-q b)} F\left(q^{i-1}\right)+z^{i} T(b) F\left(q^{i} b\right) .
$$

Iteration of the recurrence leads to

$$
F(b)=\left(z+\frac{\left(y_{i}-1\right) z^{i}}{(q ; q)_{i-1}} F\left(q^{i-1}\right)\right) \sum_{n \geqslant 0} \frac{z^{n i}}{1-q^{1+n i} b} \prod_{k=0}^{n-1} T\left(q^{k i} b\right) .
$$

Substitute $b=q^{i-1}$ and solve for $F\left(q^{i-1}\right)$ to obtain

$$
z+\frac{\left(y_{i}-1\right) z^{i}}{(q ; q)_{i-1}} F\left(q^{i-1}\right)=z\left(1-\frac{y_{i}-1}{(q ; q)_{i-1}} \sum_{n \geqslant 1} \frac{z^{n i}}{1-q^{n i}} \prod_{k=1}^{n-1} T\left(q^{k i-1}\right)\right)^{-1} .
$$

Substitute into (32) and set $b=1$ to obtain the final result.

\subsection{CCPs by peaks}

The generating functions for column-convex polyominoes by various upper ridge patterns, area, width, and relative height are always rational functions. Theorem 8 provides an example. Let $\operatorname{pic}(Q)$ be as in Corollary 11.

Theorem 8. The generating function for CCPs by peaks, area, and column number is

$$
\sum_{Q \in \mathrm{CCP}} y^{\operatorname{pic}(Q)} q^{\text {area } Q} z^{\operatorname{col} Q}=\frac{\left(\frac{z q}{1-q}+\frac{2 z^{2} q^{3}}{(1-q)^{3}}\right)\left(1+\frac{2 z q}{(1-q)^{2}}\right)}{\left(1-\frac{z q^{2}}{(1-q)^{2}}\right)\left(1+\frac{z q}{(1-q)^{2}}\right)-\frac{2 y z^{2} q^{3}}{(1-q)^{4}}} .
$$

Proof. Define $F(b)=\sum_{Q \in \mathrm{CCP}} y^{\mathrm{pic}(Q)} q^{\text {area } Q} z^{\operatorname{col} Q} h^{\text {relh } Q} b^{\alpha(Q)}$, where $\alpha(Q)$ is the area of the last column in $Q$. Let $F_{0}(b)$ be the restriction of $F(b)$ to $Q$ such that $\operatorname{col} Q \geqslant 2$ and the last pair of columns form an upper level or ascent, and let $F_{1}(b)$ be the restriction to the rest. Then, we seek $F(1)=F_{0}(1)+F_{1}(1)$, with $h=1$. As usual, we derive equations that $F_{0}$ and $F_{1}$ must satisfy:

$$
\begin{aligned}
& F_{0}(b)=\frac{z q b}{(1-q b)(1-q b h)}\left(F_{0}(1)+F_{1}(1)\right), \\
& F_{1}(b)=\frac{z q b h}{1-q b h}+\frac{z q b}{(1-q b)(1-h)}\left(y h F_{0}(1 / h)-y F_{0}(1)+h F_{1}(1 / h)-F_{1}(1)\right) .
\end{aligned}
$$

Substituting $b=1$ and $b=1 / h$ into the above system gives four equations in the four unknowns $F_{0}(1), F_{1}(1), F_{0}(1 / h)$, and $F_{1}(1 / h)$. Solve the system, compute $F_{0}(1)+F_{1}(1)$, and set $h=1$. 


\section{References}

[1] D. André, Mémoire sur les permutations alternées, J. Math. 7 (1881) 167-184.

[2] R. Angeles, D. P. Rawlings, L. Sze, and M. Tiefenbruck, The expected variation of random bounded integer sequences of finite length, Inter. J. Math. and Math. Sciences 14 (2005) 2277-2285.

[3] M. Bousquet-Mélou and X. G. Viennot, Empilements de segments et q-énumération de polyominos convexes dirigés, J. Combin. Theory (A) 60 (1992) 196-224.

[4] M. Bousquet-Mélou, A method for the enumeration of various classes of columnconvex polygons, Discrete Math. 154 (1996) 1-25.

[5] L. Carlitz, Generating functions for a special class of permutations, Proc. Amer. Math. Soc. 47 (1975) 251-256.

[6] L. Carlitz, Enumeration of compositions by rises, falls, and levels, Math. Nachr. 77 (1977), 361-371.

[7] L. Carlitz, Up-down and down-up partitions, Studies in Foundations and Combinatorics Vol. 1 (1978) 101-129.

[8] L. Carlitz and R. Scoville, Enumeration of permutations by rises, falls, rising maxima, and falling maxima, Acta Mathematica Hungarica 25 (1974) 269-277.

[9] L. Carlitz and R. Scoville, Generating functions for certain types of permutations, J. Comb. Theory Series A 18 (1975) 262-275.

[10] M. P. Delest, Polyominoes and animals: Some recent results, J. Math. Chem. 8 (1991) 3-18.

[11] M. P. Delest and J. M. Fédou, Enumeration of skew Ferrers diagrams, Discrete Math. 112 (1993) 65-79.

[12] M. P. Delest, J. P. Dubernard, I. Dutour, Parallelogram polyominoes and corners, J. Symbolic Comp. 20 (1995) 503-515.

[13] S. Elizalde and M. Noy, Consecutive patterns in permutations, Adv. Appl. Math. 30 (2003) 110-125.

[14] R. Entringer, Enumeration of permutations of $(1, \ldots, n)$ by number of maxima, Duke Math. J. 36 (1969) 575-579.

[15] J. M. Fédou, Fonctions de Bessel, empilements et tresses, in Séries Formelles et Combinatoire Algébrique, eds. P. Leroux et C. Reutenauer, Publ. du LaCIM, Unversité à Montréal, Québec 11 (1992) 189-202.

[16] I. M. Gessel, Generating Functions and Enumertation of Sequences, Doctoral Thesis, MIT, Cambridge, Massachussetts, 1977.

[17] I. P. Goulden and D. M. Jackson, An inversion theorem for cluster decompositions of sequences with distinguished subsequences, J. Lond. Math. Soc. (2) 20 (1979) $567-576$.

[18] I. P. Goulden and D. M. Jackson, Combinatorial Enumeration, Wiley, 1983.

[19] Anthony J. Guttmann, Ed. Polygons, Polyominoes and Polycubes - Lecture Notes in Physics, Vol. 775, 2009 
[20] S. Heubach and T. Mansour, Enumeration of 3-letter patterns in compositions, Combinatorial Number Theory in Celebration of the 70-th Birthday of Ronald Graham, 2007, 243-264, de Gruyter.

[21] S. Heubach and T. Mansour, Counting Rises, Levels and Drops in Compositions, Integers: Electronic Journal of Combinatorial Number Theory 5 (2005) A11.

[22] S. Heubach, S. Kitaev, T. Mansour, Partially ordered patterns and compositions, Pure Mathematics and Applications 17 No. 1-2 (2007) 1-12.

[23] S. Kitaev, Generalized patterns in words and permutations, Ph. D. thesis, Chalmers University of Technology and Göteborg University 2003.

[24] S. Kitaev, Partially ordered generalized patterns, Discrete Math. 298 (2005) 212-229.

[25] S. Kitaev, Introduction to partially ordered patterns, Discrete Applied Mathematics 155 (2007), 929-944.

[26] S. Kitaev and T. Mansour, Partially ordered generalized patterns and k-ary words, Annals of Combinatorics 7 (2003), 191-200.

[27] P. A. MacMahon, Combinatory Analysis Vol. I,II, $3^{r} d$ ed.,Chelsea Publ. Co., New York, New York, 1984.

[28] T. Mansour, Enumeration of words by the sum of differences between adjacent letters, Discrete Math. and Theoretical Comp. Science 11:1 (2009) 173-186.

[29] A. Mendes and J. B. Remmel, Permutations and words counted by consecutive patterns, Adv. Appl. Math. 374 (2006) 443-480.

[30] A. Mendes, J. B. Remmel, A. Riehl, Permutations with $k$-regular descent patterns, Elec. J. Comb., to appear.

[31] J. Noonan and D. Zeilberger, The Goulden-Jackson cluster method: Extensions, applications and implementation, J. Difference Eq. Appl. 5 (1999), 355-377.

[32] H. Prodinger and T. Tshifhumulo, On q-Olivier functions, Ann. Comb. 6 (2002) 181-194.

[33] D. P. Rawlings, Restricted words by adjacencies, Discrete Math. 220 (2000) 183-200.

[34] D. P. Rawlings, The q-exponential generating function for permutations by consecutive patterns and inversions, J. Comb. Theory Series A 114 (2007) 184-193.

[35] J. van Rensburg, The statistical mechanics of interacting walks, polygons, animals and vesicles. - Oxford University Press, 2000- (Oxford Lecture Series in Mathematics and its applications).

[36] R. P. Stanley, Enumerative Combinatorics Vol. I, Wadsworth and Brooks/Cole, Monterey, Ca. 1996.

[37] H. N. V. Temperley, Combinatorial problems suggested by the statistical mechanics of domains and rubber-like molecules, Phys. Rev. 103 (1956) 1-16.

[38] M. Tiefenbruck, Enumerating compositions with bounded parts by variation, REU report, California Polytechnic State University, San Luis Obispo, California 2003.

[39] X. G. Viennot, A Survey of Polyominoes Enumeration, SFCA Proceedings, Montréal (1992) 399-420. 